\section{REVISTA}

MEXICANA DE

ECONOMÍA Y

$\underset{\text { Nueva Epoca }}{\text { FINANZAS }}$

REMEF

(THE MEXICAN JOURNAL OF

ECONOMICS AND FINANCE)
Revista Mexicana de Economía y Finanzas

Nueva Época

Volumen 15 Número 1, Enero - Marzo 2020, pp. 57-80

DOI: https://doi.org/10.21919/remef.v15i1.403

fundación de investigación

(Recibido: 4/junio/2019, aceptado: 20/septiembre/2019)

\title{
Efecto Pass-Through en México en condiciones \\ de alta y baja volatilidad
}

Jorge Ignacio Rodríguez Carranza ${ }^{1}$

El Colegio de la Frontera Norte, México

Leticia Hernández Bielma ${ }^{2}$

El Colegio de la Frontera Norte, México

Belem Iliana Vásquez Galán ${ }^{3}$

El Colegio de la Frontera Norte, México

\section{Resumen}

El objetivo de este trabajo es medir el efecto Pass-Through en condiciones de alta y baja volatilidad cambiaria del peso-dólar sobre los precios de la Cadena Distributiva de Bienes en México. Se estimaron dos vectores autorregresivos con corrección de error y funciones de impulso-respuesta con datos mensuales de 2000 a 2017. Los resultados de las elasticidades de traspaso acumuladas indican qué, el Pass-Through genera un mayor cambio porcentual en los precios del productor, consumidor e importador en condiciones de alta volatilidad. Esto implica que el Banco de México podría instrumentar algunas medidas atenuantes a través de la política monetaria y cambiaria. Falta profundizar en las diferencias en velocidad y magnitud de traspaso a la $C D B$, lo que implica analizar la influencia de condiciones estructurales. Este trabajo destaca que el impacto en el traspaso del tipo de cambio ha sido substancialmente mayor para los precios al productor, quienes en un segundo orden lo traspasan a los precios del consumidor. Se puede concluir que el grado de volatilidad tiene un papel prominente sobre el nivel de traspaso.

Clasificación JEL: F31, E31, E52, C32

Palabras clave: Tipo de cambio, Precios, Pass-Through, Volatilidad

\section{Pass-Through Effect in Mexico in Condition of Low and High Volatility}

\section{Abstract}

This work aims to measure the Pass-Through Effect (PTE), in conditions of low and high volatility, of the peso-dollar exchange rate on the prices of the distribution channel in Mexico. Using monthly data from 2000 to 2017, two vector error correction equations and impulse response functions were estimated. The results indicate that the accumulated Pass-Through elasticity generates, in conditions of high volatility, a greater

\footnotetext{
${ }^{1}$ Carret. Esc. Tijuana - Ensenada, Km 18.5, Tijuana, Baja California, Email: jrodriguezmea2016@colef.mx

${ }^{2}$ Carret. Esc. Tijuana - Ensenada, Km 18.5, Tijuana, Baja California. Email: leticiah@colef.mx

${ }^{3}$ Técnicos 277, Monterrey, Nuevo León. Email: belem@colef.mx

* Sin fuente de financiamiento declarada para el desarrollo de la investigación
} 


\section{Abstract}

percentage change on the prices to the producer, consumer, and importer. The implications are that, through monetary and exchange rate policies, the Bank of Mexico could implement mitigation measures to reduce the PTE. Further analysis is needed on the influence of structural conditions in order to understand the differences in speed and magnitude of the PTE on the distribution channel. The impact on the exchange-rate Pass-Through has been substantially higher on the prices to the producer, who then pass on these costs to the consumers. In conclusion, the degree of volatility significantly affects how currency depreciation impacts prices.

JEL Classification: F31, E31, E52, C32

Keywords: Exchange rate, Prices, Pass-Through, Volatility

\section{Introducción}

Las crisis financieras y las depreciaciones que generan, causan incertidumbre por el posible desencadenamiento de escaladas inflacionarias, ya que el tipo de cambio actúa como ancla nominal de la inflación. La literatura económica ha consignado que existe una relación directa entre el tipo de cambio con los precios internos de bienes y servicios, conocido como efecto traspaso del tipo de cambio o "Pass-Through" (PT). Sin embargo, una depreciación no necesariamente se traduce en un incremento de la inflación. La magnitud del efecto PT depende de las condiciones macroeconómicas, microeconómicas e institucionales imperantes en la economía.

Es importante analizar los determinantes del nivel de PT, puesto que un menor nivel de traspaso del tipo de cambio permite a la autoridad bancaria central una mayor independencia en el manejo de su política monetaria. En el caso de México, el análisis del PT es muy importante dado el contexto de la política de objetivo inflacionario (POI). El objetivo de este artículo es demostrar empíricamente para el caso de la economía mexicana que, el efecto PT del tipo de cambio sobre la cadena de distribución de precios del (i) importador, (ii) productor y (iii) consumidor ${ }^{5}$, está determinado por el nivel de volatilidad cambiaria. Y que, en períodos de alta volatilidad, los precios presentan un mayor grado de traspaso cambiario. Se parte de la hipótesis de que, mediante un canal directo, la depreciación del peso incrementa los precios de los bienes de consumo importados y los costos en moneda extranjera. Por lo que, se podría esperar que los productores sean los más afectados ante las variaciones del tipo de cambio. El período de análisis es de 2000 al 2017 , período en que la economía mexicana bajo un régimen de tipo de cambio flexible ${ }^{6}$, y en un contexto de alta integración a la economía estadounidense, se ha visto afectada de manera prolongada por las grandes crisis financieras de principios del siglo XXI y por la política monetaria de los Estados Unidos.

Para determinar el efecto PT sobre la Cadena Distributiva de Bienes $(C D B)^{7}$ se estima un modelo de Vectores con Corrección de Error VEC y las Funciones Impulso-Respuesta (FIR) para medir el efecto del tipo de cambio peso-dólar sobre la $C D B$. Los resultados proveen evidencia empírica del efecto traspaso sobre los precios al importador, consumidor y productor y también muestran que el traspaso tiende a ser más intenso cuando existe mayor volatilidad cambiaria. Por lo que, este trabajo aporta en la demostración de que la mayor volatilidad contribuye a que la magnitud y velocidad del efecto traspaso sea mayor y particularmente para los precios al productor, pues los agentes reaccionan ante un escenario de alta incertidumbre. En términos de

\footnotetext{
${ }^{4}$ En español se traduce como efecto traspaso y se refiere a traspasar el aumento de costos a los precios.

${ }^{5}$ Representados por el Índice General de Precios al Importador (IGPI), el Índice Nacional de Precios al Productor (INPP) e Índice Nacional de Precios al Consumidor (INPC).

${ }^{6}$ En 1994 fue la última gran crisis que experimentó la economía mexicana bajo un régimen de tipo de cambio fijo.

${ }^{7}$ Representados por el Îndice General de Precios al Importador (IGPI), Índice Nacional de Precios al Productor (INPP) e Índice Nacional de Precios al Consumidor $(I N P C)$.
} 
la respuesta acumulada, podemos decir que en promedio el índice de precios al productor es 4.7 veces mayor al modelo de baja volatilidad, la del importador 4.1 veces y la del consumidor 7 veces mayor.

Este trabajo, aparte de la introducción, se integra de cuatro apartados. En el primero, se hace una breve revisión de la literatura; en el segundo, se expone la metodología; en el tercero, se muestran los resultados, y en el cuarto se exponen las conclusiones.

\section{El efecto Pass-Through}

\subsection{Antecedentes}

Existe una amplia literatura que analiza el comportamiento de las fluctuaciones del tipo de cambio y en particular como éste afecta a los precios internos. Miller (2003) resalta que independientemente de los choques internos de la oferta y la demanda nacional, los precios pueden verse afectados por choques externos, como las fluctuaciones del tipo de cambio, que pueden distribuirse en los tres niveles de precios en la $C D B$. Un choque cambiario se puede analizar por los efectos de primer y segundo orden de traspaso. Los efectos de primer orden ocurren por medio de un canal directo, en una primera etapa se aprecia un ajuste en los precios de bienes de consumo importados, ya sean insumos o bienes de capital que afectan de forma directa las estructuras de costos de los agentes productores. En un segundo plano, los agentes productores traspasan hacia el consumidor final el incremento en la estructura de costos por medio de un aumento de precios. Los efectos de segundo orden ocurren a través de un canal indirecto, donde el traspaso se da por medio de los mecanismos que inciden sobre la demanda agregada y que son trasladados a los precios finales vía la curva de oferta (Miller, 2003). De acuerdo a Stulz (2007) los efectos de segundo orden dependen de las expectativas y la credibilidad de los agentes económicos, si estos tienen confianza en las decisiones de la autoridad monetaria habrá una estabilidad en los precios y las presiones inflacionarias pueden ser menores. Sin embargo, bajo un ambiente de incertidumbre, los agentes pueden modificar sus expectativas y revisar sus planes de inversión, por lo que se ajustaran los precios al alza, generando presiones inflacionarias. El efecto $P T$ se evalúa considerando las tres características consignadas en la literatura, la magnitud, la velocidad y la simetría del coeficiente: 1) la magnitud mide la proporción de traspaso del ajuste en el tipo de cambio sobre los precios, y dado que varía con el tiempo, el análisis se efectúa en el corto y en el largo plazo. El nivel de traspaso puede ser completo o incompleto. El PT completo se da, si el traspaso es exactamente igual a la variación del tipo de cambio. El $P T$ incompleto se da, cuando el traspaso es menos que proporcional a dicha variación; 2) la velocidad indica el período de tiempo en que ocurre un traspaso completo a los precios debido

a un choque del tipo de cambio; y, 3) la simetría hace referencia a la respuesta de los precios ante variaciones del tipo de cambio, el coeficiente de traspaso puede ser simétrico o asimétrico. El PT simétrico indica que el traspaso a los precios es proporcional tanto para una depreciación como una apreciación, independientemente si se trata de un choque nominal o real, de demanda o de oferta, y o del ciclo económico. El PT asimétrico indica que no es proporcional y el nivel de traspaso es variable (Miller, 2003).

En la década de los ochenta, a raíz de la depreciación de la mayoría de las divisas del mundo ante el dólar estadounidense, se generó un interés particular en entender los canales de transmisión de un choque en el tipo de cambio hacia los precios. En un primer momento, la literatura económica se concentró en analizar, a nivel micro, la respuesta de los precios ante movimientos exógenos del tipo de cambio nominal. Dornbusch (1985) y Krugman (1986) realizaron los primeros análisis sobre el papel de la estructura del mercado y el comportamiento de las empresas en la fijación de precios para explicar el traspaso incompleto. La definición de esta relación entre el nivel del tipo de cambio y los precios internos de los bienes y servicios, tipificó el efecto PT. Dornbusch (1985) recurrió a los modelos de organización industrial para explicar el ajuste de los precios 
en términos de concentración de mercado. Krugman (1986), por su parte, demostró que en el caso particular de Alemania la depreciación no afectaba los precios de importación, sugiriendo cierto nivel de rigidez ante las variaciones del tipo de cambio. Krugman adjudicó este hecho al fenómeno conocido como "pricing to market" o fijación de precios al mercado, en el cual las empresas absorbían los choques externos a su estructura de costos con tal de no afectar su cuota de mercado, esta hipótesis apoyaba la plausibilidad de un PT incompleto en términos de un mercado imperfecto. Goldberg and Knetter (1996) también encontraron evidencia de un $P T$ incompleto, el cual atribuyeron a un traspaso de tercer grado con discriminación de precios. En un análisis para Australia, Dwyer et al (1993), a través de un modelo de corrección de errores (UECM), encontraron que en el corto plazo los precios de importación respondían de forma significativa a las variaciones del tipo de cambio, mientras que los precios al exportador respondían de manera retrasada, lo que podría implicar que el traspaso se absorbía en la cadena de distribución.

El auge del monetarismo habría de tener un papel importante en el enfoque de la problemática. Sobre la base de que en el largo plazo la inflación es un fenómeno monetario, los trabajos sobre el tipo de cambio y los precios pasaron de un enfoque micro a uno macro (Blundell-Wignall, 1992). McCarthy (1999) considera que este enfoque dio la oportunidad de analizar el efecto traspaso con herramientas macroeconométricas de series de tiempo. Una de las ventajas de estos modelos es que, es posible considerar la endogeneidad del tipo de cambio y permite estimar el traspaso a un conjunto de precios simultáneamente. De acuerdo con lo anterior, McCarthy (1999) estimó el nivel de PT para un grupo de economías desarrolladas ${ }^{8}$ a través de un modelo de vectores autorregresivos (VAR), posteriormente correlacionó sus resultados con algunos determinantes como: la volatilidad del PIB, del tipo de cambio, el nivel de importaciones y la competitividad. McCarthy encontró que, en el corto plazo la volatilidad del tipo de cambio y del PIB tenían una relación positiva y significativa para explicar el nivel de PT; mientras que, el nivel de importaciones también se correlacionaba con un mayor PT, pero el grado de competitividad se relacionaba de forma negativa con el PT. A partir de esta propuesta, los modelos estructurales de VAR tomaron gran popularidad para analizar el PT a nivel macro. Autores como Hahn (2003), Choudhri et al (2005), y Stulz (2007), entre otros, adoptarían esta metodología para estimar el coeficiente de PT.

En la década de los noventas muchas economías experimentaron grandes depreciaciones de sus monedas, sin embargo, el grado de $P T$ hacia los precios fue menor al de décadas pasadas. El declive en el grado de $P T$ se atribuía a un cambio estructural del entorno inflacionario, ya que también hubo una adopción generalizada de políticas monetarias con objetivo inflacionario (POI). Los trabajos posteriores llevaron a analizar otros determinantes de índole macroeconómica que explicaran el grado de PT. Gagnon \& Ihrig (2004) centraron el análisis del PT conforme a la hipótesis de Taylor (2000), este afirma que un entorno de inflación baja y estable, propicia la disminución del efecto PT. Mediante un modelo macroeconómico para veinte países industrializados y subperíodos denominados de "alta y baja inflación", los autores encontraron evidencia robusta y significativa de una relación positiva entre el grado de $P T$ y el nivel de inflación. De igual manera, Bailliu y Fuji (2004) atribuyeron una disminución en la magnitud del coeficiente de $P T$ a la estabilización del entorno inflacionario. Utilizando una metodología de panel de datos para once economías industrializadas y dos subperíodos de entorno de baja y alta inflación, encontraron evidencia empírica que respaldaba lo hallazgos de Gagnon \& Ihrig (2004).

Paralelamente a la adopción de una política de objetivo de inflación, los regímenes de tipo de cambio fijo fueron sustituidos por un régimen cambiario flexible ( $\mathrm{RCF})$. Anteriormente, el tipo de cambio fijo se utilizaba como una forma de disminuir los niveles de inflación a través de gravámenes implícitos en los precios de exportación (Edwards, 2006). Edwards obtuvo evidencia empírica para países emergentes que sustentaba la idea de que, en general, las economías con políticas de tipo de cambio flexible habían experimentado una

\footnotetext{
${ }^{8}$ EE. UU., Japón, Alemania, Francia, Reino Unido, Bélgica, Holanda, Suecia y Suiza.
} 
disminución en la magnitud del PT. Ca' Zorzi et al. (2007) contrastó el grado de PT en doce economías emergentes de Asia, Latinoamérica y Europa del Este, contra economías desarrolladas, mediante un modelo VAR, y encontró que la magnitud del coeficiente de traspaso fue menor en las economías con inflación de "un solo dígito", confirmando la relación positiva entre nivel de inflación y magnitud del coeficiente de traspaso. Estos resultados contrastan con los de Goldfajn y Werlang (2000), quienes encontraron evidencia de que el PT solía ser menor en economías desarrolladas, y mayor en países emergentes. Paralelamente a los resultados anteriores, en el caso de Suiza, Stulz (2007) también comprobó la hipótesis de Taylor (2000).

En los diversos trabajos empíricos que se han desarrollado, tanto para economías en vías de desarrollo como desarrolladas, encuentran que la hipótesis de Taylor (2000) se cumple en lo general; sin embargo, en lo que se refiere a otros determinantes del PT, los resultados han diferido. Por ejemplo, en lo que se refiere a la volatilidad del tipo de cambio y su impacto sobre la magnitud del PT, Mann (1989) indica que, para el caso de las empresas estadounidenses, ni la tendencia del movimiento del dólar ni su volatilidad, afectan los precios de las empresas. Pero, en el caso de las empresas japonesas, esto si ocurre cuando existe alta volatilidad del yen-dólar. En este caso, Miller (2003) sustenta que la relación entre la volatilidad del tipo de cambio con respecto a la magnitud del coeficiente de $P T$ es inversa, puesto que los productores y los importadores tienden a ser más cautelosos al modificar sus precios. En la medida que los empresarios afrontan costos de menú, modificarán sus precios si el beneficio de hacerlo supera estos costos. Así, a mayor volatilidad menor efecto PT (Miller, 2003: 6). De acuerdo con esto, Wei y Parsley (1995) muestran como la volatilidad del tipo de cambio hace a las firmas más adversas a cambiar sus precios, reduciendo por lo tanto el PT. Sin embargo, Mishkin y Savastano (2001) señalan que, para América Latina, el traspaso puede ser mayor debido a que las fluctuaciones de los tipos de cambio tienen un mayor efecto en la demanda y oferta agregada. Devereux y Engel (2002), por su parte, consideran que la percepción de los agentes sobre la volatilidad influirá sobre el PT, ya que, si perciben la volatilidad como permanente, el traspaso será mayor, a que si la conciben como transitoria.

En el caso de México, diversos autores han analizado el efecto PT en la economía. Ocegueda et al. (2011) investigaron si el efecto $P T$ difería entre el período con régimen de tipo de cambio fijo y con régimen flexible, encontrando que el impacto en los precios fue menor con un régimen de tipo de cambio flexible. Pérez (2012) comprobó la hipótesis de Taylor para México al contrastar los períodos previo y posterior a la adopción de la Política de Objetivo de Inflación en 1999, los resultados indican que el PT tiende a reducirse cuando existen objetivos de inflación. En estudios más recientes se ha analizado el coeficiente de traspaso hacia los distintos componentes del índice de precios al consumidor, como el de Cortés (2013) que sugiere que el PT sobre el $I N P C$ es bajo y no significativo; sin embargo, cuando analiza a nivel más desagregado, encuentra que, para el componente de precios de las mercancías no alimentarias, el coeficiente de traspaso fue positivo y significativo. Esto indica que los bienes comerciables y de importación son más susceptibles al traspaso al estar en función del mercado internacional. Castillo et al. (2013) encontraron que las características socioeconómicas específicas de cada región del país determinan la magnitud de PT, concluyendo que en el corto plazo cada región presenta distintos niveles de traspaso, mientras que en el largo plazo son muy similares.

Cuevas y Daza (2012) analizan mediante dos modelos econométricos de datos en panel, el efecto $P T$ de las variaciones cambiarias sobre los precios y la producción para cuatro países de América Latina, entre ellos México, para el período 2000-2011. En lo que se refiere a los efectos sobre la producción encontraron que la depreciación puede desestimularla por el encarecimiento de las importaciones o estimularla mediante el abaratamiento de las exportaciones, generando una inflación por costos, o una inflación por la demanda respectivamente, lo que dependerá del grado de apertura económica, aunque sugieren que este efecto debe analizarse con mayor profundidad. Verificaron que el PT del tipo de cambio a los precios "disminuye conforme la economía transita de una coyuntura de inflación elevada a otra de inflación reducida" (puesto que) (...) 
cuando la inflación es baja, resulta más sencillo para el Banco Central controlar las expectativas inflacionarias" (Cuevas y Daza, 2012, p. 207). Ellos demuestran que una depreciación de una determinada magnitud generará una mayor inflación por costos cuando la política monetaria es restrictiva, por lo que concluyen que la política monetaria influye a través del canal de costos sobre el traspaso cambiario. En este sentido, se ha visto que conocer el grado de traspaso del tipo de cambio es esencial para evaluar la efectividad de la política monetaria. Por ejemplo, Guerra (2017) sostiene que cuando el nivel de $P T$ es relativamente bajo, la autoridad monetaria puede permitir un ambiente de alta volatilidad del tipo de cambio y a la vez controlar la inflación de precios al consumidor. En el caso de México, donde el PT es incompleto, Guerra considera que el Banco Central no debería aplicar medidas de estabilización del tipo de cambio.

En un estudio reciente, Kochen y Sámano (2016), examinan el efecto PT hacia los precios al consumidor, mediante el uso de micro datos del INPC con una muestra de bienes y servicios del $58.6 \%$ del IPC para el caso de México. Su análisis abarca las implicaciones del ciclo económico en el grado de traspaso. Estos autores encuentran que existe una relación positiva entre el efecto PT y el ciclo económico, y que el traspaso del tipo de cambio a los precios al consumidor es bajo, destacando que existe heterogeneidad del efecto del $P T$ en los componentes principales del IPC.

Solorzano (2017) por su parte, también mediante el uso de micro datos y datos agregados examina si las diferencias en las tasas del efecto traspaso del tipo de cambio están asociadas con características específicas de las regiones y/o del producto. Y encuentra que las tasas del PT difieren entre regiones e industrias. Es un análisis que al distinguir el efecto a nivel de regiones e industrias permite conocer de manera desagregada la reacción de las industrias a las perturbaciones de costos. De manera que con la demostración en la diversidad de las respuestas de los precios a las perturbaciones cambiarias abre posibilidades más cibladas en materia de política monetaria.

En la actualidad la política de objetivo de inflación (POI) del Banco de México ha estado presentando niveles de inflación fuera del rango fijado en $3 \% \pm 1 \%$. Y también, la depreciación del peso frente al dólar ha llegado a niveles récord, la combinación de ambos factores podría indicar que la prolongada volatilidad a la que está expuesta la economía podría influir en el coeficiente de traspaso. Autores como Baharumshah et al. (2017) han encontrado que desde que el banco de México adopto una política de control inflacionario en 2001, el efecto $P T$ se ha debilitado sustancialmente. Por ejemplo antes de la adopción de metas de inflación, el efecto $P T$ sobre los precios era de $0.69 \%$, y con el control inflacionario disminuyo a $0.11 \%$. Este trabajo busca aportar a la literatura analizando el efecto $P T$ bajo condiciones de alta y baja volatilidad cambiaria, ya que hasta el momento no se ha identificado un análisis formal de la volatilidad del tipo de cambio y su relación con el PT, además de los efectos sobre tres índices de precios: el del productor, importador y consumidor.

\subsection{Hechos estilizados sobre la volatilidad del tipo de cambio en México}

$\mathrm{Al}$ analizar el contexto de volatilidad del tipo de cambio para México se pueden identificar tres momentos claves durante el período 2000 a 2017, altamente supeditados al comportamiento de la economía estadounidense, en los cuales el tipo de cambio nominal refleja las mayores fluctuaciones: los años 2008-2009 (con la crisis sub-prime), el año 2015 (con normalización de la política monetaria de los Estados Unidos) y los años 2016-2017 (efecto Trump y renegociación del TLCAN). Mientras, los índices de precios al productor, consumidor y de importaciones tuvieron una tendencia creciente, pero sin fluctuaciones sobresalientes (ver figura 1).

La crisis Sub-Prime del 2008, crisis hipotecaria que llevó al sistema financiero estadounidense a problemas de liquidez, generó una profunda recesión y provocó una crisis financiera global. De manera que, a finales de 2008, el consumo privado y la inversión experimentaron su mayor caída de las últimas dos décadas, esto se 
reflejó en una contracción del PIB real de Estados Unidos de América (EUA) de 6.9\% de 2007 a 2009.

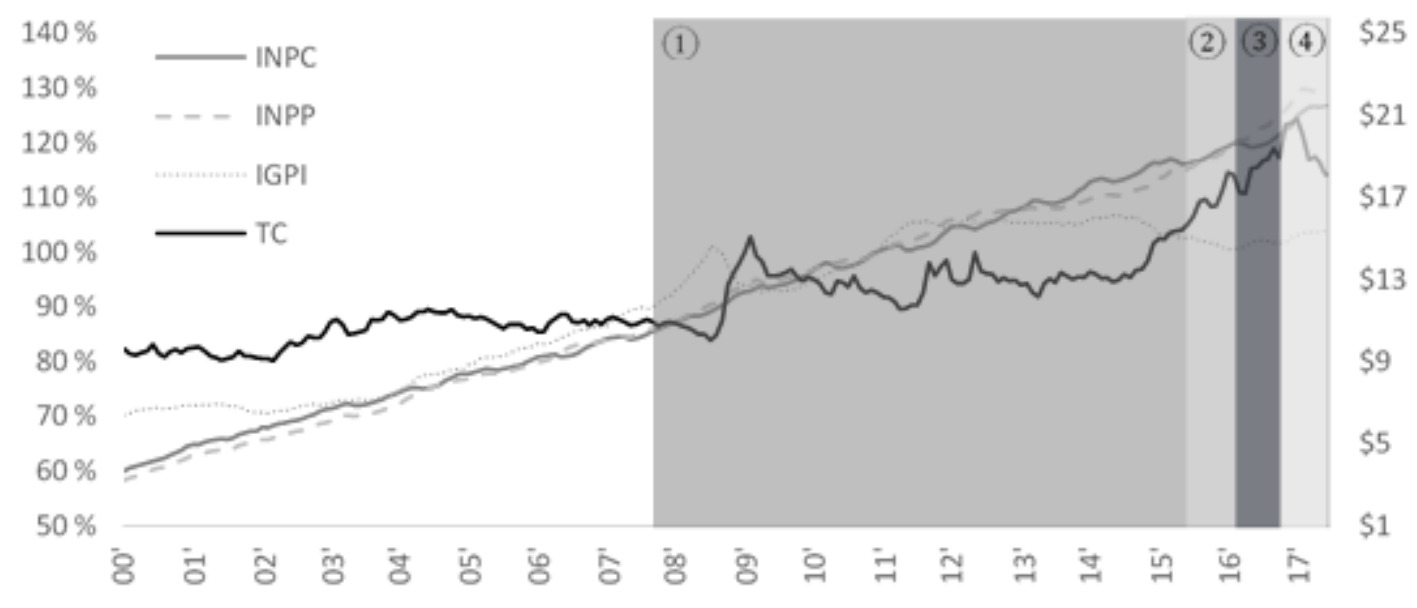

Figura 1. Tipo de cambio nominal índices de precios.

Nota: Índices de precios y tipo de cambio en niveles.

${ }^{1}$ Crisis subprime, ${ }^{2}$ normalización de tasas en EUA, ${ }^{3}$ Brexit y ${ }^{4}$ efecto "Trump".

Fuente/ elaboración propia con datos de INEGI y Banxico, 2017.

En este contexto, la crisis subprime, de acuerdo a Cuevas (2013), se transmitió a la economía mexicana mediante: la caída de las exportaciones manufactureras; la disminución de las remesas; el descenso de la Inversión Extranjera Directa; la contracción de la demanda de crudo; y las restricciones crediticias y los menores flujos de capital. (Cuevas, 2013: 15) "Estos choques externos negativos confluyeron con problemas estructurales en el ámbito doméstico" que redujeron "la eficacia de las medidas contra-cíclicas"" (Cuevas, 2013: 28). La Reserva Federal (Fed) buscó contrarrestar la crisis financiera mediante una política monetaria expansiva, medida que habrían de seguir todas las economías. El Banco de México frenó su política monetaria restrictiva, suspendiendo los incrementos a las tasas de interés a partir de septiembre de 2008 (Banxico, 2008).

En lo que respecta a la normalización de la política monetaria en EUA en 2015, esta propició un período de alta volatilidad en los mercados financieros internacionales, lo que se reflejó en una salida de capitales del mercado financiero mexicano ${ }^{9}$. El resultado fue una depreciación del tipo de cambio, lo cual, sumado al adverso contexto de precios bajos del petróleo y de bajo crecimiento global, incidió sobre las expectativas alcistas de los precios. El Banco de México motivado por los incrementos de las tasas de interés en EUA, instrumentó una política monetaria restrictiva, e incrementó la tasa de interés de referencia para evitar la fuga de capitales que pudiera representar una presión adicional al tipo de cambio, y con ello una escalada inflacionaria.

El "Efecto Trump"10 y la renegociación del Tratado de Libre Comercio de América del Norte (TLCAN) causaron, de 2016 a 2018, una depreciación del peso de $13.42 \%$, situándose en 20.78 pesos por dólar, superior a la depreciación alcanzada con el efecto Brexit y la crisis subprime. El anuncio, en mayo de 2017, de la renegociación del TLCAN, generó un mayor ambiente de incertidumbre y especulación que afectó el flujo de capitales de la economía mexicana, y la estabilidad del tipo de cambio.

\footnotetext{
${ }^{9}$ Este fenómeno se conoce como fuga hacia la calidad (flight to quality), donde en períodos de alta volatilidad los inversionistas sustituyen inversiones consideradas como riesgosas por inversiones más seguras.

${ }^{10}$ La elección de Donald Trump en noviembre de 2016.
} 


\section{Metodología}

Tomando en consideración que a lo largo del período 2000 a 2017, los eventos externos han ocasionado que las fluctuaciones del tipo de cambio peso dólar tengan diferentes grados de intensidad, se espera que el efecto traspaso a los precios en la economía nacional dependa de ese nivel de fluctuación o volatilidad cambiaria. Con base en el procedimiento metodológico aplicado por Rodríguez (2018); primero, se determina el grado de volatilidad cambiaria entre 2000 y 2017 para identificar el año que separa a un período de baja y alta volatilidad; segundo, se describe la metodología para estimar un modelo de series de tiempo que mida el efecto $P T$ del tipo de cambio sobre los precios al productor, consumidor e importador bajo dos escenarios: uno de baja volatilidad y otro de alta volatilidad.

Para identificar el cambio estructural en la volatilidad del tipo de cambio, experimentado a partir del año 2000, se emplea un modelo $G A R C H^{11}$, el cual es uno de los modelos financieros más utilizados para medir la volatilidad de las variables debido a que mejora la especificación del $A R C H$ añadiendo la varianza condicional rezagada. Esto permite calcular la volatilidad actual, además de incluir las volatilidades pasadas (Argáez, 2014). El modelo GARCH general se define de la siguiente forma:

$$
Y_{t}=\alpha+\beta Y_{t-1}+u_{t}
$$

Donde: $Y_{t}$ es el tipo de cambio diario en el período t. $Y_{t-1}$ es el tipo de cambio con un rezago. $u_{t}$ es un término de error en el período t.

La volatilidad se estima como:

$$
\sigma_{t}^{2}=w+c u_{t-1}^{2}+\delta \sigma_{t}^{2}
$$

Para modelar la volatilidad del tipo de cambio peso-dólar con una periodicidad diaria, del 3 de enero de 2000 al 30 de junio de 2017, se utilizó el modelo $\operatorname{GARCH}(1,1)$ debido a que este modelo presentó un buen ajuste, además de satisfacer los criterios de información Akaike (AIC) y Hannan-Quinn (HQ).

$$
\begin{gathered}
y_{t}=\varepsilon_{t} \text { donde } \varepsilon_{t} \mid \Psi_{t-1} N\left(0, h_{t}\right) \\
v_{t}=\frac{\varepsilon_{t}}{\sqrt{h_{t}}} \\
h_{t}=\alpha_{0}+\alpha_{1} \varepsilon_{t-1}^{2}+\beta_{1} h_{t-1}
\end{gathered}
$$

Los resultados de la estimación del modelo se presentan en el cuadro 1. Los tres coeficientes fueron significativos al $99 \%$ de nivel de confianza.

Cuadro 1. Parámetros estimados para el modelo GARCH $(1,1)$

\begin{tabular}{|c|c|c|c|}
\hline Parámetro & Coeficiente & Desv. Est. & Probabilidad \\
\hline$\alpha_{0}$ & $3.72 \mathrm{E}-07$ & $6.10 \mathrm{E}-08$ & $0.0000^{*}$ \\
\hline$\alpha_{1}$ & 0.091816 & 0.004525 & $0.0000^{*}$ \\
\hline$\beta_{1}$ & 0.902887 & 0.004948 & $0.0000^{*}$ \\
\hline
\end{tabular}

Un análisis de la varianza condicional y la desviación estándar indican que, en el período de 2000:01 a 2008:09 la volatilidad fue relativamente baja y estable, mientras que en el período 2008:10 a 2017:06, la volatilidad se incrementó considerablemente. Las mayores fluctuaciones coinciden con factores de índole

${ }^{11}$ El modelo Generalized Autoregressive Conditional Heteroskedasticity (GARCH) es introducido por Bollerslev (1986). 
externa como los ya mencionados: la crisis hipotecaria sub-prime (2008), la normalización de la política monetaria en EUA (2015), y el efecto "Trump" (2016-2017).

En la figura 2 se presenta la volatilidad cambiaria a lo largo del período 2000 a 2017, se puede ver gráficamente que la mayor volatilidad se presenta a partir de octubre del año 2008.

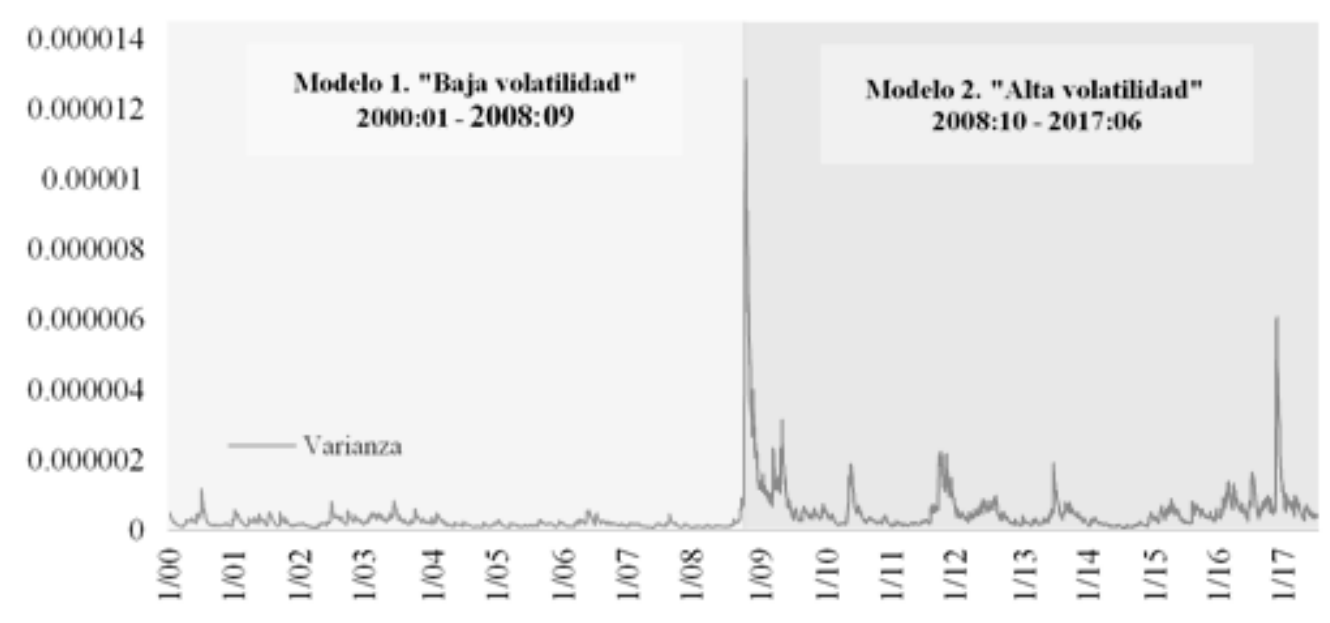

Figura 2. Volatilidad del tipo de cambio nominal peso-dólar

Fuente: Elaboración propia con datos de Banxico, 2018.

Para corroborar el cambio estructural en la volatilidad alrededor del 2008 se empleó la prueba Breakpoint Unit Root (Perron, 1989) en primera instancia se realizó la prueba anterior sobre la varianza de la volatilidad del tipo de cambio resultante del modelo $G A R C H$. Con un estadístico prueba igual a -16.46 y probabilidad menor a 0.01 con el criterio de mínimo estadístico ADF, la prueba indica un cambio estructural en octubre de 2008. Para reforzar los resultados anteriores, se aplicó la misma prueba sobre la variable tipo de cambio, en la cual el mínimo estadístico ADF fue de -24.53 y probabilidad menor a 0.01, indicando un quiebre estructural en noviembre de 2008.

Las figuras obtenidas en ambas pruebas demuestran un cambio estructural a finales de 2008, específicamente durante octubre-noviembre, por lo que estos resultados evidencian que durante el período de 2000:01 a 2008:09, la volatilidad fue relativamente baja y estable, mientras que en el período 2008:10 a 2017:06, ocurrió un cambio estructural en las variables analizadas, robusteciendo la selección de los periodos "alta y baja volatilidad".

Variable: varianza del tipo de cambio

Fecha del quiebre: $2008 / 10$

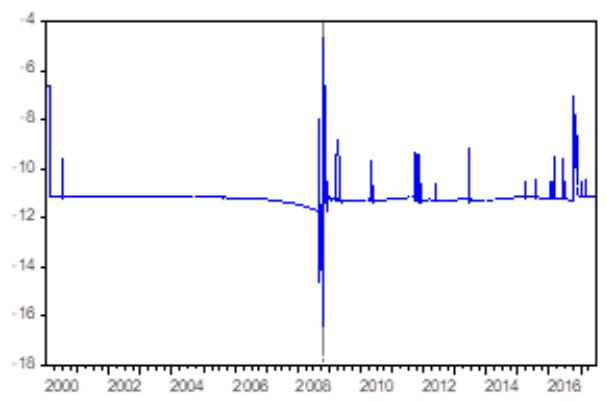

Variable: tipo de cambio Fecha del quiebre: $2008 / 11$

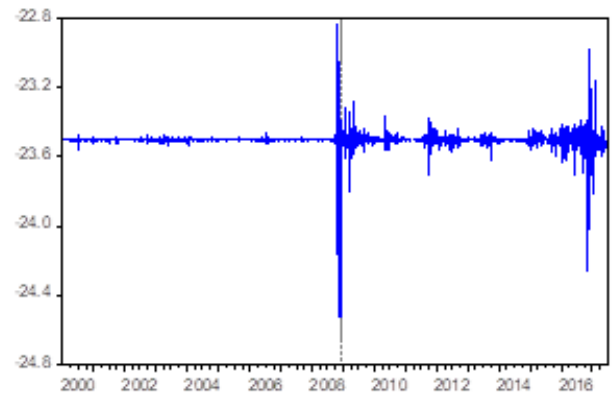

Figura 3. Estadísticos ADF de la prueba Breakpoint Unit Root.

Fuente: Elaboración propia con datos del Banco de México, 2018. 
En la literatura se identifican diferentes metodologías para medir el efecto $P T$ sobre los precios. Por ejemplo, Capistrán, et al. (2011: 4) estiman el coeficiente del PT a través de "una regresión móvil para la tasa de inflación anual contra la tasa de depreciación anual del tipo de cambio, una constante y un rezago para la inflación"12. Sin embargo, esta metodología tiene como desventaja que el modelo "no considera las interacciones tanto del tipo de cambio como de la inflación con otras variables macroeconómicas como la actividad económica o la tasa de interés" (Capistrán, et al. 2011: 5). Estudios como los de McCarthy (1999) marcaron la pauta al introducir un análisis de vectores autorregresivos o VAR, el cual tiene la ventaja de que si puede considerar las variables macroeconómicas que son determinantes para economías pequeñas y abiertas, así como analizar el efecto de los choques al tipo de cambio de forma condicionada. ${ }^{13}$

Autores como Hahn (2003), Gagnon e Ihrig (2004), Bailliu y Fujii (2004), Choudhri et al. (2005), y Stulz (2007) han adaptado los modelos VAR para estimar el traspaso de los movimientos del tipo de cambio hacia la inflación, lo que ha generado popularidad de su uso en este tipo de análisis. Para el caso mexicano dicha estrategia fue adaptada por Capistrán et al. (2012) y Cortés (2013), sin embargo, dichos trabajos fueron cuestionados por Hernández (2015: 191) por contener algunos errores metodológicos. El primer error "consiste en utilizar para la estimación de los modelos VAR datos mensuales de tasas de crecimiento anual". Dicho error conlleva a que "al acumular tasas de depreciación (inación) anual a lo largo de un cierto número de meses consecutivos no es posible obtener la depreciación acumulada (inación acumulada)". El segundo error metodológico es "el error de cálculo que resulta en un importante sesgo a la baja en la estimación de la elasticidad de traspaso del tipo de cambio a los precios", (Hernández, 2015, p. 195). Para finalizar, concluye con lo siguiente: "En términos de política económica las consecuencias son de lo más importante. La Junta de Gobierno del Banco de México ha utilizado sistemáticamente durante varios años estimaciones erróneas del traspaso del tipo de cambio a los precios para tomar decisiones de política monetaria" (Hernández, 2015: 195). Siguiendo las metodologías de McCarthy (2000), Hahn (2003), Choudhri, et al. (2005), Stulz (2007), Capistrán et al. (2012), Cortés (2013) y Rodríguez (2015), así como incorporando las observaciones de Hernández (2015), se especificó un modelo de vectores autorregresivos que permite analizar la dinámica entre las perturbaciones que afectan al tipo de cambio y a los precios, controlado por la dinámica de otras variables macroeconómicas. Formalmente el modelo de vectores autorregresivos (VAR) se expresa de la siguiente forma:

$$
Y_{t}=\pi_{1} Y_{t-1}+\ldots+\pi_{p} Y_{t-p}+\pi X_{t}+e_{t}
$$

Donde: $Y_{t}$ es un vector de $\mathrm{k}$ variables endógenas. $X_{t}$ es un vector de variables exógenas. $\pi_{1}, \ldots, \pi_{2}$ y $\pi$ son matriz de coeficientes y $e_{t}$ es un vector de innovaciones.

Las seis variables endógenas del sistema de ecuaciones son: los índices de precios del productor (INPP), del consumidor $(I N P C)$, de las importaciones (IGPI), la tasa de interés del certificado de la tesorería (Cete) a 28 días (TI), el índice global de la actividad económica (IGAE) y el tipo de cambio (TC). Las cuatro variables exógenas son: el índice de producción industrial de EUA (IPI), la tasa de bonos del tesoro de los EUA (R), el índice de precios al consumidor de EUA (IPC) y el índice de precios internacionales de las materias primas del FMI (IC). Todas las variables están en frecuencia mensual, fueron deflactadas con año base 2010 y están en logaritmos naturales. ${ }^{14}$

El modelo 1 a estimar se denomina de "Baja volatilidad" y comprende el período de enero del 2000 a septiembre del 2008 (contiene 105 observaciones). Y, el modelo 2 se denomina de "Alta volatilidad" y comprende de octubre del 2008 a junio del 2017 (contiene 105 observaciones). Uno de los desafíos de la estimación de un

\footnotetext{
${ }^{12}$ Utilizada por Capistrán et al. (2011:5).

${ }^{13}$ Controlado por las otras variables que pudieran afectar a los precios.

${ }^{14} \mathrm{La}$ descripción detallada de las variables se encuentra en el anexo 1. También fueron convertidas a logaritmos naturales. Las fuentes de información proceden del INEGI, Banco de México, Reserva Federal de los EUA y del Fondo Monetario Internacional.
} 
$V A R$ es la selección del nivel óptimo de rezagos, para lo cual se ha establecido que el modelo seleccionado debe satisfacer la condición de estabilidad matemática, de homocedasticidad y de correlación serial. Por lo tanto, se aplicaron las pruebas de estabilidad matemática, White y LM, así como la de normalidad Jarque-Bera.

Las funciones de impulso-respuesta se calculan a partir de las estimaciones del VAR o VEC y miden la respuesta de cada variable endógena a un impulso en las innovaciones estructurales. Por lo tanto, los efectos del PT se miden por medio del cociente de los impulsos-respuesta acumulados de un choque hacia los precios sobre los impulsos-respuesta acumulados de un choque en el tipo de cambio, que se identifican mediante el método recursivo (utilizando la descomposición de Cholesky) ${ }^{15}$. La fórmula para calcular la elasticidad de traspaso acumulada es:

$$
P T_{\tau}=\frac{\Delta \% P_{t, t+\tau}}{\Delta \% T C_{t, t+\tau}}
$$

Donde: $\mathrm{PT}_{\tau}$ es la elasticidad acumulada en el período $\tau . \Delta \% P_{t, t+\tau}$ es el cambio porcentual en los precios $\tau$ períodos después del choque y $\Delta \% T C_{t, t+\tau}$ es el cambio porcentual en el tipo de cambio $\tau$ períodos después del choque. El resultado también se puede interpretar como el cambio porcentual en los índices de precios ante una depreciación de $1 \%$ del tipo de cambio.

La estimación con series de tiempo requiere conocer el grado de integración de las variables y si están o no cointegradas. Por lo tanto, a las variables de nuestro modelo se les aplicaron las pruebas de raíz unitaria Augmented Dickey-Fuller (ADF), Phillips-Perron (PP) y KPSS para determinar el grado de integración. Los resultados de estas pruebas indicaron que todas las series son estacionarias en primeras diferencias (ver anexo 2), por lo tanto, fue necesario aplicar una prueba de cointegración.

La prueba de cointegración nos dice si existe una relación de largo plazo entre variables no estacionarias, y de ser así, identifica el número de relaciones de cointegración. Si no existe cointegración se puede estimar el modelo (6) en primeras diferencias, de lo contrario se tiene que incluir un mecanismo de corrección de error y estimar un vector (VEC) como el siguiente:

$$
\Delta y_{t}=\Gamma y_{t-1}+\pi_{1} \Delta y_{t-1}+\ldots+\pi_{p-1} \Delta y_{t-p+1}+\pi X_{t}+e_{t}
$$

Donde $\Gamma y_{t-1}$ contiene las relaciones de cointegración y representa el parámetro de largo plazo. $\Delta$ indica primeras diferencias.

Para determinar si existe cointegración, se aplicó la prueba sugerida por Johansen-Juselius (1991) ${ }^{16}$. Se estimaron dos modelos VAR para tomar en cuenta el período de Baja Volatilidad (modelo 1) y el de Alta Volatilidad (modelo 2). La selección óptima de los rezagos para ambos modelos satisfizo las condiciones de estabilidad matemática, homocedasticidad, normalidad y correlación serial. Por lo tanto, la prueba de Johansen se aplicó a un $\operatorname{VAR}(7)$ para el modelo "1" y a un $V A R(3)$ para el modelo 2. Los resultados se presentan en el cuadro 2 e indican que, en ambos modelos, las variables endógenas están cointegradas.

\footnotetext{
${ }^{15}$ Procedimiento utilizado por los autores Capistrán et al., 2012.

${ }^{16}$ La metodología de Johansen (1991) está orientada a complementar la especificación de un VEC, partiendo de la matriz de coeficientes $\pi$ de la ecuación $Y_{t}=v+\pi Y_{t-1}+e_{t}$ que representa el modelo VAR, al estimar dicha matriz, si alguno de estos coeficientes resulta ser igual a uno, esto implica un rango reducido de la matriz $\pi$, por lo tanto, existe una combinación lineal de las variables incluidas en el sistema que es estacionaria, es decir existe un vector de cointegración.
} 
Cuadro 2. Resultados de la prueba de cointegración de Johansen

\begin{tabular}{|c|c|c|c|c|c|c|}
\hline \multicolumn{7}{|c|}{ Modelo 1. "Baja Volatilidad"2000:01 a 2008:09 } \\
\hline $\begin{array}{l}\text { Hipótesis } \\
\text { nula }\end{array}$ & Traza & $\begin{array}{c}\text { Valor } \\
\text { crítico } 95 \%\end{array}$ & Probabilidad & Max-Eig & $\begin{array}{c}\text { Valor } \\
\text { crítico } 95 \%\end{array}$ & Probabilidad \\
\hline$r \geq 0^{*}$ & 131.299 & 95.754 & 0 & 50.838 & 40.078 & 0.002 \\
\hline$r \geq 1^{*}$ & 80.461 & 69.819 & 0.0056 & 27.533 & 33.877 & 0.236 \\
\hline$r \geq 2 *$ & 52.928 & 47.856 & 0.0155 & 23.984 & 27.584 & 0.135 \\
\hline$r \geq 3$ & 28.944 & 29.797 & 0.0625 & 18.348 & 21.132 & 0.117 \\
\hline$r \geq 4$ & 10.596 & 15.495 & 0.2376 & 7.742 & 14.265 & 0.406 \\
\hline \multicolumn{7}{|c|}{ Modelo 2. . ${ }^{\mathrm{A}}$ lta Volatilidad"2008:10 a 2017:06 } \\
\hline $\begin{array}{l}\text { Hipótesis } \\
\text { nula }\end{array}$ & Traza & $\begin{array}{c}\text { Valor } \\
\text { crítico } 95 \%\end{array}$ & Probabilidad & Max-Eig & $\begin{array}{c}\text { Valor } \\
\text { crítico } 95 \%\end{array}$ & Probabilidad \\
\hline$r \geq 0^{*}$ & 175.164 & 95.754 & 0 & 60.79 & 40.078 & 0 \\
\hline $\mathrm{r} \geq 1^{*}$ & 114.373 & 69.819 & 0 & 54.94 & 33.877 & 0 \\
\hline $\mathrm{r} \geq 2 *$ & 59.433 & 47.856 & 0.003 & 38.147 & 27.584 & 0.002 \\
\hline$r \geq 3$ & 21.287 & 29.797 & 0.34 & 12.893 & 21.132 & 0.462 \\
\hline $\mathrm{r} \geq 4$ & 8.394 & 15.495 & 0.424 & 7.352 & 14.265 & 0.448 \\
\hline
\end{tabular}

Los resultados indican que los dos modelos se deben estimar como vectores autorregresivos con corrección de error (VEC), lo que implica que tenemos una parte de las estimaciones que describen la relación de largo plazo entre las variables y otra parte que describe las relaciones de corto plazo.

Una prueba que nos permite medir la relación de corto plazo que existe entre el tipo de cambio y los diferentes índices de precios y nos dice la dirección de causalidad entre ellas, es la de Granger. Mediante esta prueba es posible saber cuáles incrementos de precios están precedidos por un aumento del tipo de cambio.

\section{Análisis de resultados}

Los modelos VEC especificados en (8) fueron estimados con siete rezagos para el modelo 1 de baja volatilidad y con dos rezagos para el modelo 2 de alta volatilidad. La selección de rezagos siguió los criterios de selección AIC y HQ, y además cumplieron con las condiciones de estabilidad matemática, ausencia de correlación serial, homocedasticidad y normalidad (ver los resultados de las pruebas en el anexo 3).

\subsection{Causalidad de Granger}

Los resultados de la prueba de causalidad de Granger se presentan en el cuadro 3 y muestran que la hipótesis nula de no causalidad solo se rechaza en el modelo 2. Se comprueba que en el período de volatilidad alta existe causalidad de Granger que va del tipo de cambio hacia los precios del importador, del productor y del consumidor. Podemos decir que el efecto traspaso ocurre solamente cuando existen condiciones de alta volatilidad cambiaria, es decir, la incertidumbre y las expectativas de los agentes contribuyen a que cualquier depreciación del tipo de cambio se traduzca en un aumento de precios. Por otro lado, encontramos que, con baja volatilidad, y por lo tanto mayor estabilidad cambiaria, la causalidad no es significativa. Adicionalmente, también encontramos que independientemente de que exista alta o baja volatilidad, el índice de precios al productor causa en el sentido de Granger al índice de precios al consumidor. 
Cuadro 3. Causalidad de Granger "Prueba de Wald"

\begin{tabular}{|c|c|}
\hline Hipótesis nula: & Probabilidad \\
\hline \multicolumn{2}{|c|}{ Modelo 1. Baja volatilidad VEC (7) } \\
\hline TC no causa en sentido de Granger a IGPI & 0.3485 \\
\hline TC no causa en sentido de Granger a INPP & 0.1113 \\
\hline TC no causa en sentido de Granger a INPC & 0.6640 \\
\hline \multicolumn{2}{|c|}{ Modelo 2. Alta volatilidad VEC (2) } \\
\hline TC no causa en sentido de Granger a IGPI & $0.0054^{* *}$ \\
\hline TC no causa en sentido de Granger a INPP & $0.0000^{* *}$ \\
\hline TC no causa en sentido de Granger a INPC & $0.0273^{*}$ \\
\hline
\end{tabular}

\section{2. $\quad$ El efecto Pass-Through a través del impulso-respuesta acumulado}

Con base en los modelos VEC especificados anteriormente, y por medio de la descomposición de Cholesky, se obtuvieron las funciones impulso-respuesta para cada modelo, permitiendo analizar y contrastar la magnitud y velocidad del traspaso del tipo de cambio a los índices de precios IGPI, INPC e INPP, con un horizonte de 24 meses (ver resultados en anexo 4).

En general, las funciones impulso-respuesta indican que un choque de $1 \%$ del tipo de cambio tiene mayor impacto sobre los precios de la $C D B$ en el período de alta volatilidad. En promedio, la magnitud es del doble que en el período de baja volatilidad. En cuanto a la velocidad, un choque cambiario parece causar en el corto plazo efectos mixtos, primero ocasiona una disminución temporal en los precios de importación, para posteriormente aumentarlos de manera constante a partir del tercer período, sin embargo, los precios de los agentes se estabilizan a partir del octavo mes. En contraste, con baja volatilidad, aunque el impacto es cuantitativamente menor, la fluctuación de los precios no parece estabilizarse. En especial, los precios a las importaciones (IGPI) mantienen una respuesta fluctuante, de alzas y bajas a lo largo del horizonte de veinticuatro meses. Este fenómeno fue percibido por Ocegueda et al (2011, p. 130), quienes afirman que "es importante destacar la necesidad de nuevas investigaciones que clarifiquen lo ocurrido en el período de tipo de cambio flexible en el cual se registró una menor influencia en el esquema de transmisión de precios de la economía y en donde se obtuvo un efecto negativo de las fluctuaciones de los precios de las importaciones sobre los precios internos." A priori, de acuerdo con la evidencia empírica internacional, se esperaba una mayor respuesta en los precios a las importaciones $(I G P I)$, no obstante, para el caso de la economía mexicana esto no sucede. Al parecer las empresas importadoras absorben estos choques de tipo de cambio y reducen los precios en el corto plazo, siempre y cuando se mantenga una certidumbre en el tipo de cambio, es decir, una baja volatilidad. Estos hallazgos refuerzan la hipótesis de que la volatilidad cambiaria es un determinante importante en la estabilidad de los precios para una economía como la mexicana, contrario a otras economías desarrolladas.

Una visión más completa la ofrecen las funciones de impulso respuesta acumuladas, que permiten ver la respuesta acumulada de cada índice de precios a un choque en las innovaciones del tipo de cambio (ver anexo 5). En la figura 4 se presenta de manera gráfica la respuesta acumulada del IGPI ante innovaciones generalizadas y acumuladas de una desviación estándar, no solo del tipo de cambio (TC), sino también de los índices de precios del productor $(I N P P)$ y del consumidor $(I N P C)$, considerando los dos períodos de volatilidad. Lo primero que salta a la vista es que, con baja volatilidad, la respuesta acumulada de los precios de las importaciones es reducida ante un choque del tipo de cambio, lo que corresponde con otros hallazgos en el sentido que las empresas no ajustan sus precios a los movimientos del tipo de cambio (Betts y Devereux, 
1996). Sin embargo, lo que sí es notorio es que la respuesta de los precios al importador es mayor ante un choque del tipo de cambio cuando la volatilidad es alta.
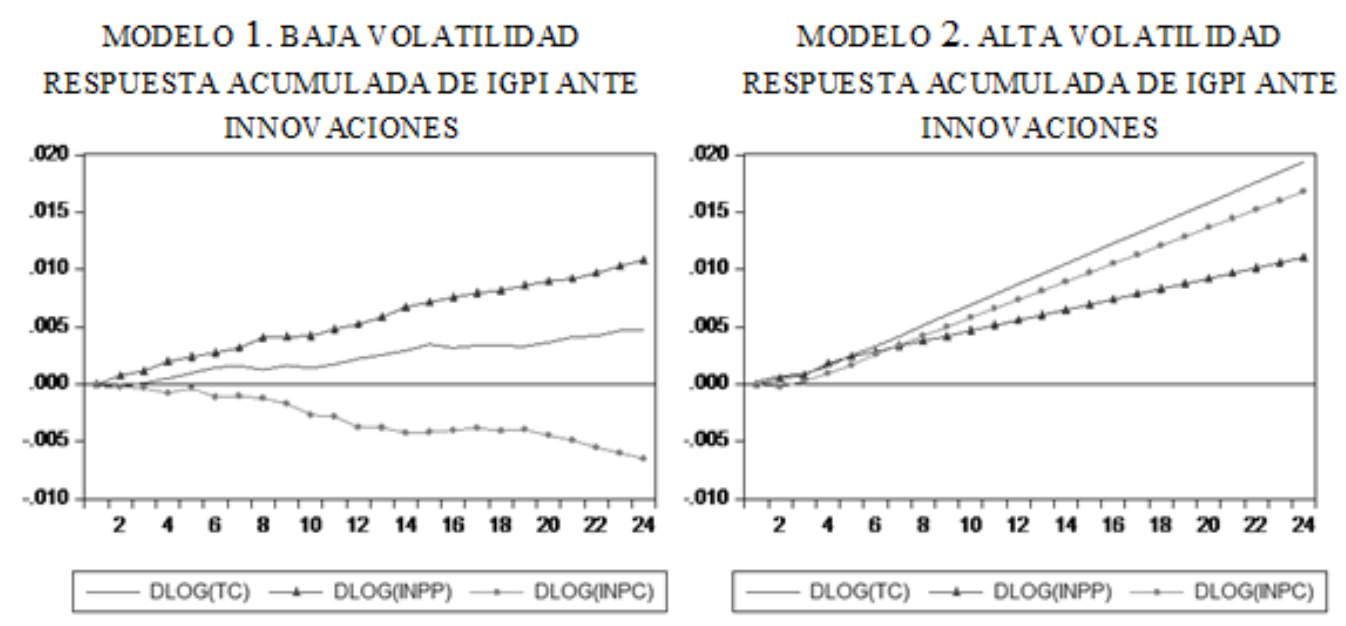

Figura 4. Respuesta acumulada de los precios al importador (IGPI)

Fuente: Elaboración propia.

En el caso de la respuesta acumulada del índice de precios al productor (INPP), ésta ocurre principalmente ante un choque del TC. En la figura 5 se puede ver que, no solo los precios al productor responden al choque del tipo de cambio, sino que la magnitud y velocidad de su respuesta es mayor en el período de alta volatilidad que en el de baja volatilidad. Además, de acuerdo con la teoría, el INPP también responde a un choque del $I G P I$. En este sentido, los coeficientes son mayores en un contexto de alta volatilidad, ya que, además del efecto traspaso del tipo de cambio, se recibe una presión adicional de los precios al importador.
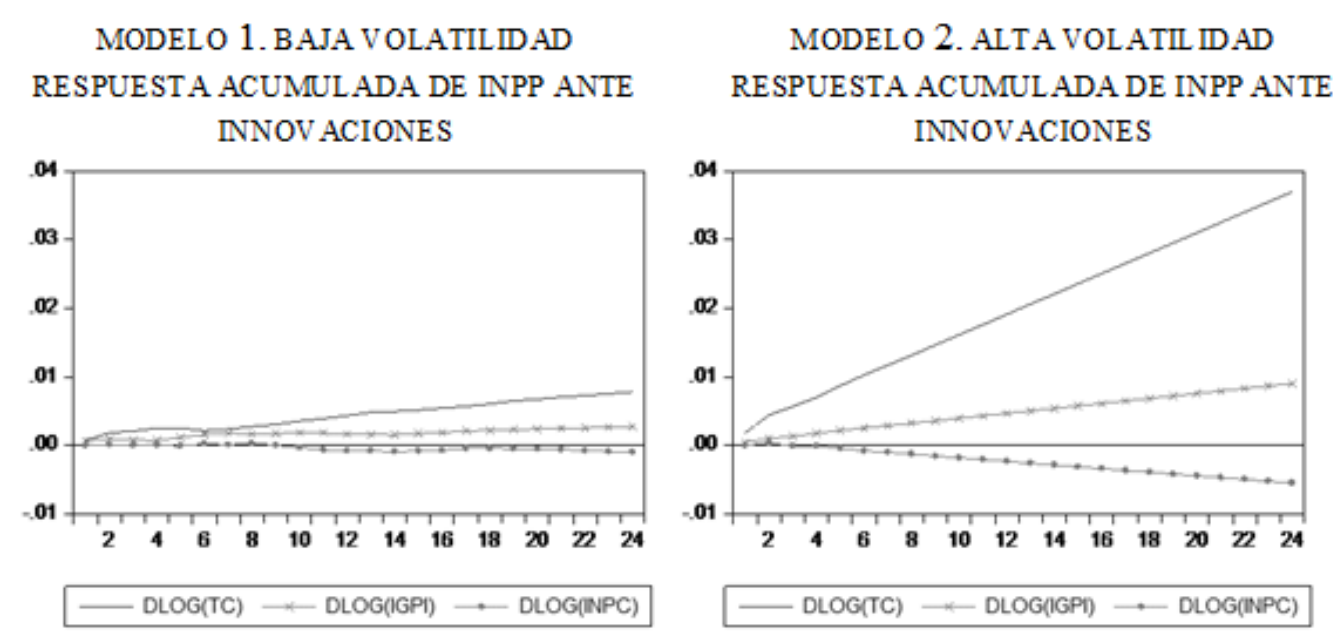

Figura 5. Respuesta acumulada del índice de precios al productor (INPP)

Fuente: Elaboración propia.

Finalmente, encontramos que el índice de precios al consumidor $(I N P C)$ responde de manera positiva a un choque del tipo de cambio, sin embargo, como se observa en la figura 6, la respuesta acumulada es reducida con baja volatilidad y aumenta en un contexto de mayor volatilidad. Cuando hay estabilidad cambiaria, los precios al consumidor parecen responder únicamente a un choque en los precios del productor, lo cual es congruente con lo que se espera cuando no se deprecia la moneda. Sin embargo, con alta volatilidad los 
precios al consumidor tienden a incrementar su respuesta acumulada a una depreciación de la moneda y a los precios a las importaciones, pero no ante los precios del productor. Una razón que podría explicar este resultado es que, los productores son los que absorben en sus estructuras de costos las variaciones del tipo de cambio. Esta hipótesis se basa en el fenómeno microeconómico de "pricing to market" y con la teoría neokeynesiana de costos de menú "price-stickiness", que sostiene que los productores absorben en primera instancia los choques del traspaso cambiario derivados de un incremento en la estructura de costos, por lo tanto, en el corto plazo se presenta rigidez de precios (Gopinath y Rigobon, 2008).
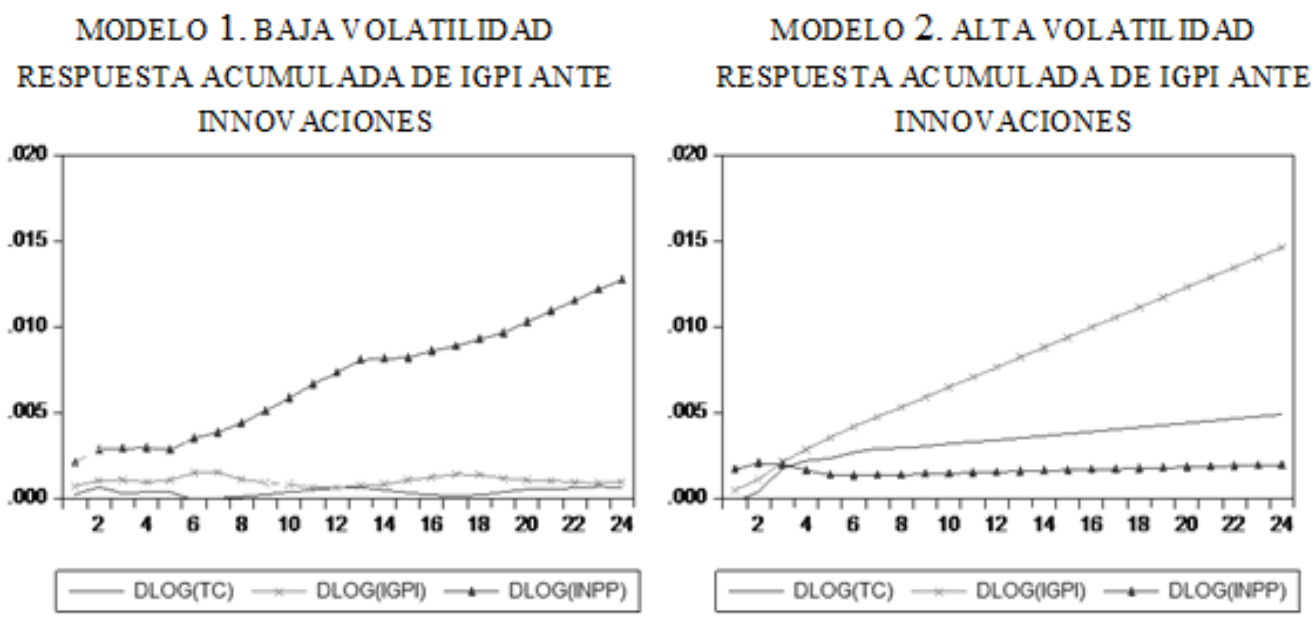

Figura 6. Respuesta acumulada del $I N P C$

Fuente: Elaboración propia.

Hasta ahora la evidencia confirma la hipótesis general de que la respuesta acumulada de los precios a un choque en el tipo de cambio es mayor, en magnitud y velocidad, en el período de alta volatilidad. Encontramos que, en promedio, el modelo de alta volatilidad estima la mayor respuesta del índice de precios al productor de hasta 4.7 veces mayor al modelo de baja volatilidad, mientras que la respuesta de los precios al importador es 4.1 veces más alta y la del consumidor es 7 veces mayor. En específico, los precios al productor (INPP) son los que reciben el mayor efecto traspaso, tanto en períodos de alta como de baja volatilidad, por lo que estos agentes son los más sensibles a las fluctuaciones del tipo de cambio.

\subsection{El efecto Pass-Through en el largo plazo}

Sobre el efecto $P T$ en el largo plazo existen diversos estudios empíricos como el de Rodríguez (2015), quien encuentra que el traspaso de primer orden para México es completo. Cruz et al. (2015) sostienen que en el largo plazo la magnitud del efecto $P T$ es igual o cercana a uno. Internacionalmente estos resultados coinciden con los hallados por Shioji (2012), Goldfajn y Werlang (2000), Dwyer y Pease (1993), y Frankel et al. (2012). De manera que, las variaciones del tipo de cambio se traspasan por completo a los precios en el largo plazo, como lo predice la Ley del Precio Único y la Teoría de Paridad de Poder Adquisitivo $(P P A)^{17}$. Debido a lo anterior, resulta imperativo saber cómo evoluciona el nivel de $P T$ (a 12 meses) y sus efectos en la cadena de distribución de bienes $(C D B)$ para el caso de México. En el cuadro 4 se presentan las elasticidades del traspaso acumulado de cada índice de precios frente a un choque en el tipo de cambio, y fueron calculadas a partir de la formula (7). Las elasticidades también se pueden interpretar como el cambio porcentual en los

\footnotetext{
${ }^{17}$ Para Krugman, el enfoque monetarista teóricamente cumple con la $P P A$.
} 
precios debido a una depreciación de $1 \%$ del tipo de cambio. Un valor igual a 1 nos indicaría un traspaso completo.

En general, encontramos que las elasticidades de traspaso a los precios son mayores en el modelo de alta volatilidad. La evidencia sugiere que los precios al productor son los más afectados en el largo plazo, recibiendo el mayor traspaso ante una depreciación de $1 \%$ del tipo de cambio (de 0.049 y $0.224 \%$ durante cada período). Esto podría explicarse debido a que, mediante un canal directo, una depreciación del peso causaría un ajuste hacia el alza en los precios de insumos importados y de otros costos como deuda o inversiones denominadas en dólares que forman parte de sus costos totales. En este contexto, el tipo de cambio tiene un efecto directo sobre la estructura de costos de los agentes productores.

La elasticidad de traspaso a los precios al consumidor tiene un efecto directo de 0.013 y 0.040 para el periodo de baja y alta volatilidad, respectivamente. En este sentido el resultado del periodo 2000 a 2008 se acerca al obtenido por Cortés (2013) quien con una muestra de 2001 a 2012 encuentra que la elasticidad de traspaso para los precios al consumidor fue de 0.03 después de 12 meses. Por otro lado, se presentan efectos indirectos cuando un cambio en los precios al importador y productor se traslada a los demás agentes de la economía. Por ejemplo, por cada incremento porcentual en los precios del productor, los precios del consumidor aumentan $0.87 \%$ y $0.13 \%$ en el periodo de baja y alta volatilidad respectivamente, generando en conjunto un aumento generalizado de precios. En el caso de los precios al importador, vemos que, con baja volatilidad, estos responden a una depreciación del tipo de cambio de manera directa con una magnitud de 0.048, mientras que, con alta volatilidad el coeficiente es de 0.102. En un segundo orden, el productor presenta un efecto traspaso indirecto similar desde los precios del importador en ambos escenarios (0.1860 contra 0.1928); sin embargo, en el caso del consumidor ante los precios del importador, estos responden con marcado efecto indirecto en el escenario de alta volatilidad (0.0751 contra 0.3159). Estos resultados coinciden con otros, que han encontrado que los precios a las importaciones son los que reciben el mayor efecto traspaso y son los que desencadenan un efecto inflacionario en la cadena de precios (Capistran et al., 2012).

Cuadro 4. Elasticidades de traspaso acumuladas del tipo de cambio hacia los índices de precios de corto plazo (horizonte a 12 meses).

\begin{tabular}{|c|c|c|}
\hline Efecto de primer & Baja volatilidad & Alta volatilidad \\
\cline { 2 - 3 } orden: TC - $>$ Índices de Precios & \multicolumn{2}{|c|}{ efecto directo } \\
\hline Importaciones $(I G P I)$ & 0.048 & $\mathbf{0 . 1 0 2}$ \\
\hline Productor $(I N P P)$ & 0.049 & $\mathbf{0 . 2 2 4}$ \\
\hline Consumidor $(I N P C)$ & 0.013 & $\mathbf{0 . 0 4}$ \\
\hline
\end{tabular}

Fuente: elaboración propia con estimaciones obtenidas con VECs.

Cuadro 5. Elasticidades de traspaso acumuladas de segundo orden (horizonte de 12 meses).

\begin{tabular}{|c|c|c|}
\hline Efecto de segundo orden: & Baja volatilidad & Alta volatilidad \\
\hline Precios - $>$ Precios & \multicolumn{2}{|c|}{ efecto indirecto } \\
\hline Importaciones $(I G P I)$ - $>$ Productor $(I N P P)$ & 0.186 & $\mathbf{0 . 1 9 2 8}$ \\
\hline Importaciones $(I G P I)$ - $>$ Consumidor $(I N P C)$ & 0.0751 & $\mathbf{0 . 3 1 5 9}$ \\
\hline Productor $(I N P P)$ - $>$ Consumidor $(I N P C)$ & $\mathbf{0 . 8 7 8 6}$ & 0.1375 \\
\hline
\end{tabular}

Fuente: elaboración propia con estimaciones obtenidas con VECs.

A partir de los resultados, contrastando ambos modelos, se puede decir que la volatilidad cambiaria prolongada genera un período de incertidumbre que provoca que los agentes perciban choques externos en el tipo de cambio como perdurables y no transitorios, lo que influye directamente en sus expectativas. En este sentido, en un primer orden, los productores presentan el mayor efecto de traspaso directo durante el período de alta volatilidad procedente del tipo de cambio; mientras que, en un segundo orden, el nivel de traspaso 
desde los precios al importador se mantiene a la par en ambos escenarios. Por otra parte, los productores tienden a trasladar este incremento de precios al consumidor, en un alto porcentaje, durante un período de baja volatilidad cambiaria. Lo anterior se encuentra en línea con la teoría neokeynesiana de costos del menú "price-stickiness", la cual indica que, los productores absorben en primera instancia los choques del traspaso cambiario derivados del aumento en la estructura de costos, por ende, en el corto plazo el efecto indirecto de traspaso al consumidor desde el productor, tiende a reducirse en períodos de incertidumbre.

\section{Conclusiones}

En la literatura ha existido, durante décadas, una amplia preocupación por el tipo de cambio en relación con la política monetaria y la dinámica de la inflación. En los últimos años, varios de estos trabajos se han centrado en el análisis del PT, y han encontrado que se ha dado una disminución de este efecto. La explicación de este resultado lo atribuyen a las políticas de corte monetarista que han prevalecido en las últimas décadas en la economía mundial, donde la política monetaria en particular se ha centrado en la llamada política de objetivo inflacionario y un régimen de tipo de cambio flexible.

México, a su vez, como parte de la tendencia mundial en materia de control monetario, ha acompañado la liberalización de su economía con transformaciones en su institución bancaria central, dándole autonomía al Banco de México, que ha fijado como único objetivo el control de la inflación, y ha abandonado el régimen del tipo de cambio fijo. Todo esto ha permitido llevar adelante una política monetaria altamente restrictiva. Sin embargo, dado que México es un pequeño país y, en el contexto de una economía abierta, altamente supeditado a la economía estadounidense en materia comercial y financiera, el control de la política monetaria no ha sido suficiente, y los precios continúan expuestos en períodos de alta volatilidad cambiaria, a sucesos económicos relacionados con nuestro principal socio comercial

De acuerdo a los resultados de este artículo encontramos que, por una parte, en un efecto de primer orden, y en línea con resultados que ya se han consignado en la literatura, las variaciones del tipo de cambio afectan más los precios al productor durante el período de alta volatilidad. Por otro lado, en un segundo orden, los precios al consumidor son los que reciben el mayor nivel de traspaso durante un escenario de alta volatilidad, adicionalmente los precios al importador mantienen una presión en condiciones de alta y baja volatilidad al productor. Por lo que, en un contexto de contracción de la demanda agregada global, la afectación a los precios tiene su origen en los costos, y no en causas monetarias, cuestión que se explica por el mismo contexto de restricción monetaria y el alto control del Banco de México sobre el circulante durante el período analizado.

La evidencia empírica es acorde con la hipótesis de Taylor (2000), pues demuestra que, con menor volatilidad, los precios son menos sensibles a los choques del tipo de cambio que cuando existe mayor volatilidad y por lo tanto incertidumbre. La volatilidad juega un papel determinante en el grado de traspaso en México. Esto puede explicar que, en un contexto de relativa estabilidad y con una política de fuerte control monetario, entre 2000 y 2007 los precios dieron lugar a un bajo nivel de traspaso debido a un mayor grado de certidumbre en el tipo de cambio. Y, por el contrario, en un contexto de aguda crisis económica iniciada a finales de 2008, la mayor incertidumbre en la perspectiva económica tanto por los factores internos como externos, dio lugar a la mayor volatilidad del tipo de cambio, por lo cual la magnitud y la velocidad del traspaso a los precios internos fue mayor. En este sentido, para este período, la presente investigación implica un nuevo acercamiento para el caso de México sobre el PT, contemplando los niveles de volatilidad cambiaría, los resultados aportan evidencia empírica sobre la influencia de la incertidumbre cambiaría sobre este fenómeno.

Las políticas monetarias de objetivo de inflación y régimen de tipo de cambio flexible, sin duda han sido efectivas en la contención de los precios, y al igual que en el resto de las economías ha contribuido a una disminución del efecto traspaso. Pero su efectividad se ha visto reducida en condiciones de alta volatilidad, 
pues no se ha podido atenuar el efecto traspaso del tipo de cambio. Lo que revela que existen condiciones estructurales en la economía mexicana que están al origen del impacto de las variaciones del tipo de cambio, y que la política monetaria, con el solo objetivo inflacionario, no puede solucionar en contextos de alta incertidumbre económica.

Las condiciones estructurales están determinadas por el modo de inserción de la economía mexicana en el mercado norteamericano. Particularmente, a partir del TLCAN se ha condicionado al aparato productivo y comercial del país hacia una estrecha interdependencia con la economía estadounidense, donde las transnacionales vía la segmentación de sus procesos productivos, han conducido a una intensificación del comercio intraindustrial, lo que ha provocado la alta dependencia de México hacia la importación de insumos y bienes de capital, lo que afecta al impacto del tipo de cambio en los precios.

Por último, se debe profundizar más en esta problemática, a partir del estudio detallado de los mecanismos de transmisión de precios de las diferentes $C D B$, sobre las razones que explican la velocidad y magnitud con la que se traslada el aumento de costos al consumidor, puesto que los productores son los que reciben el mayor efecto traspaso. Asimismo, dado que en la actualidad las nuevas tecnologías han permitido desarrollar innovadoras técnicas de análisis, donde destacan los modelos de series de tiempo dinámicos Time-varying coefficients VAR (TVC-VAR), el campo de estudio del big data puede aportar un gran avance en el análisis de este fenómeno a un mayor nivel de desagregación de la $C D B$.

\section{Anexos}

1. Descripción de las variables utilizadas en el modelo de estimación.

\begin{tabular}{|c|c|c|c|c|c|}
\hline Variable & Descripción & Unidad & Base & Frecuencia & Fuente \\
\hline$I G P I$ & Índice General de Precios de las Importaciones & Índice & $2010 \mathrm{M} 12$ & Mensual & INEGI \\
\hline$I N P P$ & Índice Nacional de Precios al Productor & Índice & $2010 \mathrm{M} 12$ & Mensual & INEGI \\
\hline$I N P C$ & Índice Nacional de Precios al Consumidor & Índice & $2010 \mathrm{M} 12$ & Mensual & INEGI \\
\hline TC & Tipo de cambio al cierre fix, peso dólar & Niveles & - & Mensual & Banxico \\
\hline IPI & Índice de producción industrial de EUA & Índice & $2010 \mathrm{M} 12$ & Mensual & FED \\
\hline R & Tasa de bonos del Tesoro de EUA a un mes & Niveles & - & Mensual & FED \\
\hline IPC & Índice de precios al consumidor de EUA & Índice & $2010 \mathrm{M} 12$ & Mensual & BLS \\
\hline IC & Índice mensual de precios internacionales de materias primas & Índice & $2010 \mathrm{M} 12$ & Mensual & FMI \\
\hline IGAE & Índice Global de la Actividad Económica & Índice & $2010 \mathrm{M} 12$ & Mensual & INEGI \\
\hline TI & Tasa de interés de Cetes a 28 días & Niveles & - & Mensual & Banxico \\
\hline
\end{tabular}


2. Resultados de las pruebas de raíz unitaria a las variables del modelo.

\begin{tabular}{|c|c|c|c|c|c|c|c|c|c|}
\hline \multicolumn{10}{|c|}{ Período 2000:01 a 2017:06 } \\
\hline Variable & Prueba & Especificación & Nivel & Prob. & Primera diferencia & Prob. & Valor crítico $1 \%$ & Valor crítico $5 \%$ & Decisión* \\
\hline \multirow{3}{*}{$\mathrm{TC}$} & $\mathrm{ADF}$ & Inter. y Tend. & -2.327 & 0.417 & -12.639 & 0.000 & -4.002 & -3.431 & \multirow{3}{*}{$\mathrm{I}(1)$} \\
\hline & $\mathrm{PP}$ & Inter. y Tend. & -2.327 & 0.417 & -12.563 & 0.000 & -4.002 & -3.431 & \\
\hline & KPSS & Inter. y Tend. & 0.165 & $\sim$ & 0.031 & $\sim$ & 0.216 & 0.146 & \\
\hline \multirow{3}{*}{$I N P C$} & $\mathrm{ADF}$ & Inter. & -0.413 & 0.903 & -3.913 & 0.002 & -3.462 & -2.875 & \multirow{3}{*}{$\mathrm{I}(1)$} \\
\hline & $\mathrm{PP}$ & Inter. & -1.944 & 0.312 & -8.058 & 0.000 & -3.462 & -2.875 & \\
\hline & KPSS & Inter. & 1.858 & $\sim$ & 0.519 & $\sim$ & 0.739 & 0.463 & \\
\hline \multirow{3}{*}{$I N P P$} & $\mathrm{ADF}$ & Inter. y Tend. & -2.517 & 0.320 & -10.815 & 0.000 & -4.002 & -3.431 & \multirow{3}{*}{$\mathrm{I}(1)$} \\
\hline & $\mathrm{PP}$ & Inter. y Tend. & -2.585 & 0.288 & -10.869 & 0.000 & -4.002 & -3.431 & \\
\hline & KPSS & Inter. y Tend. & 0.369 & $\sim$ & 0.097 & $\sim$ & 0.216 & 0.146 & \\
\hline \multirow{3}{*}{$I G P I$} & $\mathrm{ADF}$ & Inter. y Tend. & -1.388 & 0.862 & -5.420 & 0.000 & -4.002 & -3.431 & \multirow{3}{*}{$\mathrm{I}(1)$} \\
\hline & $\mathrm{PP}$ & Inter. y Tend. & -0.754 & 0.967 & -8.856 & 0.000 & -4.002 & -3.431 & \\
\hline & KPSS & Inter. y Tend. & 0.330 & $\sim$ & 0.113 & $\sim$ & 0.216 & 0.146 & \\
\hline \multirow{3}{*}{ TI } & $\mathrm{ADF}$ & Inter. y Tend. & -1.148 & 0.917 & -13.137 & 0.000 & -4.002 & -3.431 & \multirow{3}{*}{$\mathrm{I}(1)$} \\
\hline & $\mathrm{PP}$ & Inter. y Tend. & -1.690 & 0.967 & -13.187 & 0.000 & -4.002 & -3.431 & \\
\hline & KPSS & Inter. y Tend. & 0.088 & $\sim$ & 0.081 & $\sim$ & 0.216 & 0.146 & \\
\hline \multirow{3}{*}{ IGAE } & $\mathrm{ADF}$ & Inter. y Tend. & -2.127 & 0.527 & -14.965 & 0.000 & -4.002 & -3.431 & \multirow{3}{*}{$\mathrm{I}(1)$} \\
\hline & $\mathrm{PP}$ & Inter. y Tend. & -2.401 & 0.378 & -14.989 & 0.000 & -4.002 & -3.431 & \\
\hline & KPSS & Inter. y Tend. & 0.133 & $\sim$ & 0.041 & $\sim$ & 0.216 & 0.146 & \\
\hline \multirow{3}{*}{ IPI } & $\mathrm{ADF}$ & Inter y Tend. & -3.343 & 0.062 & -3.704 & 0.024 & -4.002 & -3.431 & \multirow{3}{*}{$\mathrm{I}(1)$} \\
\hline & PP & Inter y Tend. & -2.189 & 0.493 & -12.799 & 0.000 & -4.002 & -3.431 & \\
\hline & KPSS & Inter y Tend. & 0.107 & $\sim$ & 0.041 & $\sim$ & 0.216 & 0.146 & \\
\hline \multirow{3}{*}{$\mathrm{R}$} & $\mathrm{ADF}$ & None & -1.343 & 0.166 & -9.110 & 0.000 & -2.576 & -1.942 & \multirow{3}{*}{$\mathrm{I}(1)$} \\
\hline & $\mathrm{PP}$ & None & -1.289 & 0.182 & -9.110 & 0.000 & -2.576 & -1.942 & \\
\hline & KPSS & Inter. y Tend. & 0.156 & $\sim$ & 0.159 & $\sim$ & 0.216 & 0.146 & \\
\hline \multirow{3}{*}{ IPC } & $\mathrm{ADF}$ & Inter. y Tend. & -1.277 & 0.891 & -9.944 & 0.000 & -4.002 & -3.431 & \multirow{3}{*}{$\mathrm{I}(1)$} \\
\hline & $\mathrm{PP}$ & Inter. y Tend. & -1.216 & 0.904 & -8.809 & 0.000 & -4.002 & -3.431 & \\
\hline & KPSS & Inter. y Tend. & 0.384 & $\sim$ & 0.056 & $\sim$ & 0.216 & 0.146 & \\
\hline \multirow{3}{*}{$\mathrm{IC}$} & $\mathrm{ADF}$ & None & 0.357 & 0.787 & -9.620 & 0.000 & -2.576 & -1.942 & \multirow{3}{*}{$\mathrm{I}(1)$} \\
\hline & $\mathrm{PP}$ & None & -1.870 & 0.804 & -6.535 & 0.000 & -2.576 & -1.942 & \\
\hline & KPSS & Inter. y Tend. & 0.342 & $\sim$ & 0.055 & $\sim$ & 0.216 & 0.146 & \\
\hline
\end{tabular}

*Con base en las pruebas ADF y PP al $95 \%$ de confianza.

Fuente: elaboración propia con datos de INEGI, Banxico, FED, BLS y FMI, 2018.

3. Pruebas de diagnóstico aplicadas a los residuales

3.1 Estabilidad matemática.

\section{RESUL TADOS RAÍZ INVERSA DEL POLINOMIO CARACTERÍSTICO AR MODELO 1. BAJA VOLATIL IDAD MODELO 2. ALTA VOLATILIDAD}
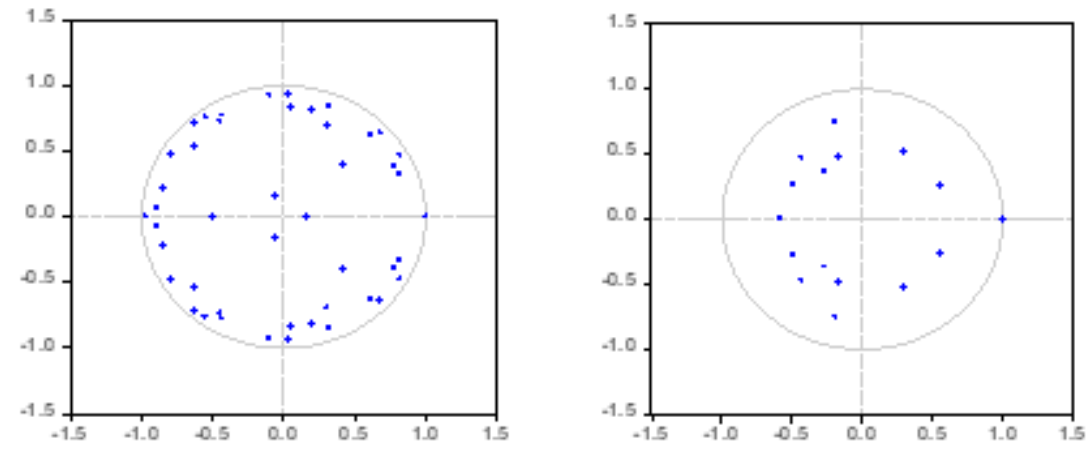
3.2 Correlación serial.

\begin{tabular}{|c|c|c|c|c|c|}
\hline \multicolumn{6}{|c|}{ RESULTADOS DE LA PRUEBA BREUSCH-GODFREY } \\
\hline \multicolumn{3}{|c|}{ MODELO 1. BAJA VOLATILIDAD } & \multicolumn{3}{|c|}{ MODELO 2. ALTA VOLATILIDAD } \\
\hline \multicolumn{3}{|c|}{ Muestra: 2000M01 2008M09 } & \multicolumn{3}{|c|}{ Muestra: 2008M10 2017M06 } \\
\hline \multicolumn{3}{|c|}{ Observaciones: 105} & \multicolumn{3}{|c|}{ Observaciones: 105} \\
\hline REZAGOS & LM-STAT & PROBABILIDAD & REZAGOS & LM-STAT & PROBABILIDAD \\
\hline 1 & 30.742 & 0.717 & 1 & 43.816 & 0.174 \\
\hline 2 & 39.164 & 0.330 & 2 & 51.490 & 0.045 \\
\hline 3 & 34.742 & 0.528 & 3 & 40.587 & 0.275 \\
\hline 4 & 26.579 & 0.874 & & & \\
\hline 5 & 33.628 & 0.582 & & & \\
\hline 6 & 33.237 & 0.601 & & & \\
\hline 7 & 24.056 & 0.936 & & & \\
\hline 8 & 31.865 & 0.666 & & & \\
\hline
\end{tabular}

3.3 Homocedasticidad.

\begin{tabular}{|c|c|c|c|c|c|}
\hline \multicolumn{5}{|c|}{ Resultados de la prueba de white } \\
\hline \multicolumn{3}{|c|}{ Modelo 1. baja volatilidad } & \multicolumn{3}{c|}{ Modelo 2. alta volatilidad } \\
\hline Muestra: 2000M01 a 2008M09 & \multicolumn{2}{c|}{ Muestra: 2008M10 a 2017M06 } \\
\hline Chi-sq & df & Prob. & Chi-sq & df & Prob. \\
\hline 2088.3 & 2058 & 0.316 & 813.521 & 798 & 0.344 \\
\hline
\end{tabular}

3.4 Normalidad

\begin{tabular}{|c|c|c|c|c|c|c|c|}
\hline \multicolumn{8}{|c|}{ Resultados de la prueba jarque-bera } \\
\hline \multicolumn{3}{|c|}{ Modelo 1. baja volatilidad } & \multicolumn{3}{c|}{ Modelo 2. alta volatilidad } \\
\hline \multicolumn{2}{|c|}{ Muestra: 2000M01 a 2008M09 } & \multicolumn{3}{c|}{ Muestra: 2008M10 a 2017M06 } \\
\hline Component & Jarque-Bera & df & Prob. & Component & Jarque-Bera & df & Prob. \\
\hline Joint & 4.799 & 12 & 0.964 & Joint & 39.774 & 12 & 0 \\
\hline
\end{tabular}

4. Funciones de Impulso-Respuesta.
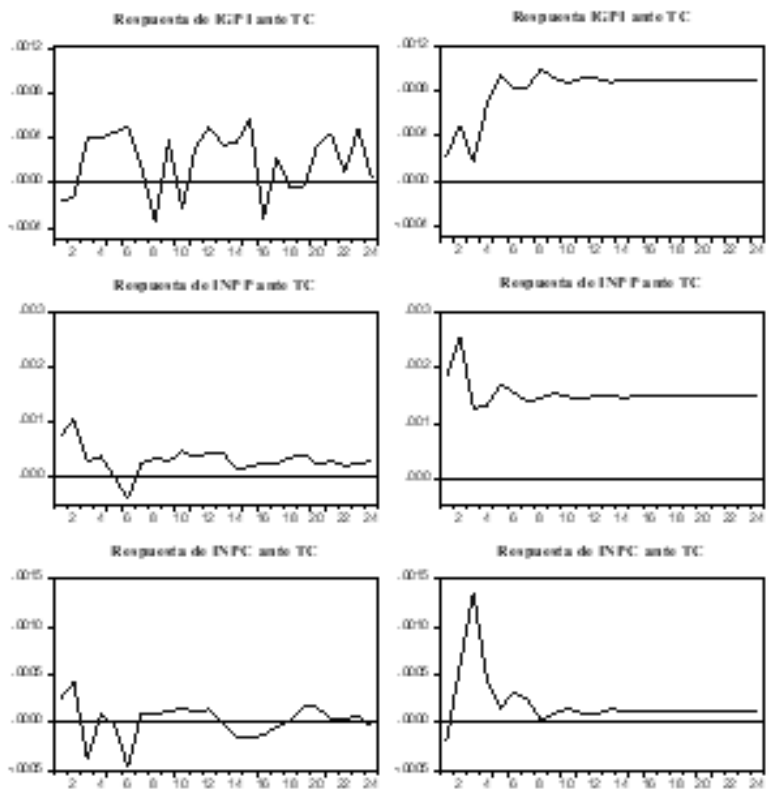

Fuente: elaboración propia con datos de INEGI, Banxico, FED, BLS y FMI, 2017. 
5. Impulso-respuesta acumuladas a un choque en el tipo de cambio.

\begin{tabular}{|c|c|c|c|c|c|c|c|c|c|c|c|c|c|c|}
\hline \multicolumn{15}{|c|}{ MODELO 1. BAJA VOLATILIDAD } \\
\hline \multicolumn{5}{|c|}{$\begin{array}{l}\text { RESPUESTA PRECIOS AL } \\
\text { IMPORTADOR IGPI }\end{array}$} & \multicolumn{5}{|c|}{$\begin{array}{l}\text { RESPUESTA PRECIOS AL } \\
\text { PRODUCTOR INPP }\end{array}$} & \multicolumn{5}{|c|}{$\begin{array}{l}\text { RESPUESTA PRECIOS AL } \\
\text { CONSUMIDOR INPC }\end{array}$} \\
\hline MES & TC & $I G P I$ & $I N P P$ & $I N P C$ & MES & TC & $I G P I$ & $I N P P$ & $I N P C$ & MES & TC & $I G P I$ & $I N P P$ & $I N P C$ \\
\hline 1 & -0.0007 & 0.0027 & 0.0000 & 0.0000 & 1 & 0.0003 & 0.0003 & 0.0022 & 0.0000 & 1 & 0.0000 & 0.0003 & 0.0018 & 0.0017 \\
\hline 2 & -0.0019 & 0.0047 & 0.0006 & -0.0006 & 2 & 0.0012 & 0.0005 & 0.0045 & -0.0001 & 2 & 0.0003 & 0.0005 & 0.0033 & 0.0034 \\
\hline 3 & -0.0033 & 0.0064 & 0.0013 & -0.0013 & 3 & 0.0022 & 0.0002 & 0.0063 & -0.0004 & 3 & 0.0006 & 0.0007 & 0.0042 & 0.0047 \\
\hline 4 & -0.0043 & 0.0080 & 0.0024 & -0.0022 & 4 & 0.0033 & -0.0003 & 0.0079 & -0.0005 & 4 & 0.0013 & 0.0005 & 0.0043 & 0.0056 \\
\hline 5 & -0.0054 & 0.0092 & 0.0032 & -0.0026 & 5 & 0.0042 & -0.0004 & 0.0091 & -0.0004 & 5 & 0.0020 & 0.0004 & 0.0038 & 0.0062 \\
\hline 6 & -0.0056 & 0.0096 & 0.0039 & -0.0035 & 6 & 0.0044 & -0.0006 & 0.0105 & 0.0001 & 6 & \begin{tabular}{|l|}
0.0020 \\
\end{tabular} & 0.0006 & 0.0037 & 0.0064 \\
\hline 7 & \begin{tabular}{|l|}
-0.0058 \\
\end{tabular} & 0.0103 & 0.0039 & \begin{tabular}{|l|}
-0.0038 \\
\end{tabular} & 7 & 0.0049 & -0.0009 & 0.0115 & 0.0003 & 7 & \begin{tabular}{|l|}
0.0022 \\
\end{tabular} & 0.0007 & 0.0036 & 0.0055 \\
\hline 8 & -0.0060 & 0.0105 & 0.0040 & -0.0045 & 8 & 0.0056 & -0.0013 & 0.0120 & 0.0002 & 8 & 0.0023 & 0.0005 & 0.0039 & 0.0041 \\
\hline 9 & -0.0060 & 0.0107 & 0.0041 & -0.0052 & 9 & 0.0065 & -0.0018 & 0.0126 & 0.0001 & 9 & \begin{tabular}{|l|}
0.0024 \\
\end{tabular} & 0.0003 & 0.0049 & 0.0028 \\
\hline 10 & \begin{tabular}{|l|}
-0.0058 \\
\end{tabular} & 0.0107 & 0.0044 & -0.0066 & 10 & 0.0080 & -0.0023 & 0.0130 & 0.0000 & 10 & 0.0026 & 0.0000 & 0.0066 & 0.0020 \\
\hline 11 & -0.0057 & 0.0108 & 0.0047 & -0.0075 & 11 & 0.0095 & -0.0028 & 0.0135 & -0.0002 & 11 & 0.0029 & -0.0005 & 0.0087 & 0.0015 \\
\hline 12 & -0.0054 & 0.0108 & 0.0055 & -0.0089 & 12 & 0.0113 & -0.0035 & 0.0138 & -0.0001 & 12 & 0.0037 & -0.0011 & 0.0107 & 0.0018 \\
\hline 13 & \begin{tabular}{|l|}
-0.0053 \\
\end{tabular} & 0.0110 & 0.0063 & -0.0097 & 13 & 0.0131 & -0.0042 & 0.0141 & 0.0002 & 13 & \begin{tabular}{|l|}
0.0047 \\
\end{tabular} & -0.0019 & 0.0123 & 0.0028 \\
\hline 14 & -0.0050 & 0.0110 & 0.0074 & -0.0104 & 14 & 0.0149 & -0.0048 & 0.0145 & 0.0009 & 14 & 0.0058 & -0.0026 & 0.0131 & 0.0043 \\
\hline 15 & -0.0045 & 0.0110 & 0.0082 & -0.0104 & 15 & 0.0167 & -0.0054 & 0.0147 & 0.0017 & 15 & 0.0072 & -0.0032 & 0.0132 & 0.0057 \\
\hline 16 & \begin{tabular}{|l|}
-0.0039 \\
\end{tabular} & 0.0109 & 0.0087 & -0.0101 & 16 & 0.0184 & -0.0060 & 0.0149 & 0.0025 & 16 & 0.0085 & -0.0037 & 0.0126 & 0.0068 \\
\hline 17 & -0.0032 & 0.0108 & 0.0087 & -0.0095 & 17 & 0.0200 & -0.0064 & 0.0147 & 0.0033 & 17 & 0.0098 & -0.0039 & 0.0115 & 0.0074 \\
\hline 18 & -0.0023 & 0.0106 & 0.0082 & -0.0089 & 18 & 0.0216 & -0.0068 & 0.0144 & 0.0038 & 18 & 0.0109 & -0.0040 & 0.0104 & 0.0074 \\
\hline 19 & -0.0014 & 0.0106 & 0.0071 & -0.0084 & 19 & 0.0231 & -0.0072 & 0.0140 & 0.0041 & 1 & 0.0117 & -0.0039 & 0.0096 & .0069 \\
\hline 20 & -0.0005 & 0.0106 & 0.0059 & -0.0083 & 20 & 0.0245 & -0.0075 & 0.0137 & 0.0042 & 20 & 0.0125 & -0.0039 & 0.0094 & 0.0063 \\
\hline 21 & 0.0003 & 0.0107 & 0.0046 & -0.0085 & 21 & 0.0256 & -0.0079 & 0.0134 & 0.0042 & 21 & 0.0131 & -0.0039 & 0.0098 & 0.0057 \\
\hline 22 & 0.0010 & 0.0108 & 0.0036 & -0.0090 & 22 & 0.0267 & -0.0083 & 0.0134 & 0.0042 & 2 & 0.0138 & -0.0041 & 0.0107 & 0.0055 \\
\hline 23 & 0.0015 & 0.0110 & 0.0030 & -0.0097 & 23 & 0.0276 & -0.0088 & 0.0135 & 0.0042 & 23 & 0.0146 & -0.0045 & 0.0117 & 0.0057 \\
\hline 24 & 0.0018 & 0.0110 & 0.0029 & -0.0103 & 24 & 0.0284 & -0.0093 & 0.0138 & 0.0044 & 24 & 0.0154 & -0.0050 & 0.0126 & 0.0065 \\
\hline \multicolumn{15}{|c|}{ MODELO 2. ALTA VOLATILIDAD } \\
\hline \multicolumn{5}{|c|}{$\begin{array}{l}\text { RESPUESTA PRECIOS } \\
\text { AL IMPORTADOR IGPI }\end{array}$} & \multicolumn{5}{|c|}{$\begin{array}{l}\text { RESPUESTA PRECIOS } \\
\text { AL PRODUCTOR INPP }\end{array}$} & \multicolumn{5}{|c|}{$\begin{array}{l}\text { RESPUESTA PRECIOS } \\
\text { AL CONSUMIDOR INPC }\end{array}$} \\
\hline MES & TC & $I G P I$ & MES & TC & $I G P I$ & MES & TC & $I G P I$ & MES & TC & IGPI & MES & TC & $I G P I$ \\
\hline 1 & -0.0006 & 0.0038 & 0.0000 & 0000 & 1 & 0.0017 & \begin{tabular}{l|l}
7 & 0.0005
\end{tabular} & 0.0024 & 0.0000 & 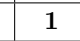 & 0.0001 & -0.0002 & 0.0016 & 0.0023 \\
\hline 2 & -0.0015 & 0.0073 & 0.0002 & -0.0011 & 2 & 0.0050 & \begin{tabular}{l|l} 
& 0.0014
\end{tabular} & 0.0043 & 0.0006 & 2 & 0.0012 & -0.0007 & 0.0034 & 0.0058 \\
\hline 3 & -0.0020 & 0.0109 & 0.0003 & -0.0019 & 3 & 0.0080 & 0.0021 & 0.0061 & 0.0010 & 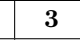 & 0.0036 & -0.0016 & 0.0049 & 0.0098 \\
\hline 4 & -0.0020 & 0.0140 & 0.0004 & -0.0027 & 4 & 0.0104 & \begin{tabular}{l|l}
4 & 0.0024 \\
\end{tabular} & 0.0079 & 0.0013 & 4 & 0.0057 & -0.0030 & 0.0064 & 0.0134 \\
\hline 5 & $\mid-0.0011$ & 0.0168 & 0.0005 & -0.0035 & 5 & 0.0124 & \begin{tabular}{|l|l}
4 & 0.0027
\end{tabular} & 0.0097 & 0.0015 & 5 & 0.0075 & -0.0049 & 0.0080 & 0.0166 \\
\hline 6 & -0.0003 & 0.0190 & 0.0005 & -0.0042 & 6 & 0.0142 & \begin{tabular}{|l|l|}
2 & 0.0029
\end{tabular} & 0.0112 & 0.0015 & 6 & 0.0091 & -0.0068 & 0.0094 & 0.0192 \\
\hline 7 & 0.0005 & 0.0207 & 0.0004 & -0.0049 & 7 & 0.0155 & \begin{tabular}{|l|l|}
5 & 0.0032 \\
\end{tabular} & 0.0126 & 0.0012 & 7 & 0.0105 & -0.0086 & 0.0108 & 0.0211 \\
\hline 8 & 0.0010 & 0.0222 & 0.0003 & -0.0056 & 8 & 0.0164 & \begin{tabular}{l|l}
4 & 0.0037 \\
\end{tabular} & 0.0138 & 0.0007 & 8 & 0.0117 & -0.0101 & 0.0123 & 0.0224 \\
\hline 9 & 0.0013 & 0.0234 & 0.0000 & -0.0064 & 9 & 0.0172 & \begin{tabular}{|l|l|}
2 & 0.0043
\end{tabular} & 0.0148 & 0.0002 & 9 & 0.0126 & -0.0113 & 0.0136 & 0.0232 \\
\hline 10 & 0.0013 & 0.0246 & -0.0004 & -0.0072 & 10 & 0.0179 & 0.0051 & 0.0156 & -0.0003 & 10 & 0.0135 & -0.0122 & 0.0149 & 0.0237 \\
\hline 11 & 0.0012 & 0.0257 & -0.0008 & -0.0080 & 11 & 0.0185 & \begin{tabular}{|l|l}
5 & 0.0059 \\
\end{tabular} & 0.0163 & -0.0007 & 11 & 0.0143 & -0.0127 & 0.0162 & 0.0239 \\
\hline 12 & 0.0009 & 0.0267 & -0.0013 & -0.0087 & 12 & 0.0192 & \begin{tabular}{l|l}
2 & 0.0069
\end{tabular} & 0.0169 & -0.0009 & 12 & 0.0152 & -0.0131 & 0.0174 & 0.0241 \\
\hline 13 & 0.0006 & 0.0277 & -0.0018 & -0.0092 & 13 & 0.0199 & \begin{tabular}{l|l}
9 & 0.0079 \\
\end{tabular} & 0.0173 & -0.0010 & 13 & 0.0161 & -0.0132 & 0.0185 & 0.0243 \\
\hline 14 & 0.0004 & 0.0287 & -0.0024 & -0.0096 & 14 & 0.0208 & \begin{tabular}{l|l}
3 & 0.0088
\end{tabular} & 0.0176 & -0.0008 & 14 & 0.0171 & -0.0132 & 0.0196 & 0.0244 \\
\hline 15 & 0.0002 & 0.0296 & -0.0030 & -0.0099 & 15 & 0.0216 & 0.0097 & 0.0178 & -0.0005 & 15 & 0.0181 & -0.0132 & 0.0205 & 0.0246 \\
\hline 16 & 0.0001 & 0.0304 & -0.0036 & -0.0099 & 16 & 0.0226 & \begin{tabular}{|l|l|}
5 & 0.0104
\end{tabular} & 0.0179 & 0.0000 & 16 & 0.0190 & -0.0131 & 0.0214 & 0.0248 \\
\hline 17 & 0.0000 & 0.0311 & -0.0043 & -0.0099 & 17 & 0.0235 & \begin{tabular}{|l|l|}
5 & 0.0111 \\
\end{tabular} & 0.0180 & 0.0005 & 17 & 0.0200 & -0.0129 & 0.0222 & 0.0250 \\
\hline 18 & 0.0000 & 0.0316 & -0.0049 & -0.0097 & 18 & 0.0244 & \begin{tabular}{l|l} 
& 0.0117
\end{tabular} & 0.0180 & 0.0012 & 18 & 0.0209 & -0.0127 & 0.0230 & 0.0252 \\
\hline 19 & 0.0000 & 0.0320 & -0.0055 & -0.0094 & 19 & 0.0252 & 0.0122 & 0.0179 & 0.0019 & 19 & 0.0217 & -0.0125 & 0.0236 & 0.0255 \\
\hline 20 & 0.0000 & 0.0323 & -0.0060 & -0.0090 & 20 & 0.0261 & \begin{tabular}{l|l}
1 & 0.0126 \\
\end{tabular} & 0.0179 & 0.0027 & 20 & 0.0225 & -0.0123 & 0.0242 & 0.0257 \\
\hline 21 & 0.0001 & 0.0325 & -0.0066 & -0.0087 & 21 & 0.0269 & \begin{tabular}{l|l}
9 & 0.0130
\end{tabular} & 0.0178 & 0.0034 & 21 & 0.0233 & -0.0120 & 0.0246 & 0.0260 \\
\hline 22 & 0.0001 & 0.0327 & -0.0071 & -0.0083 & 22 & 0.0276 & \begin{tabular}{|l|l}
5 & 0.0133
\end{tabular} & 0.0176 & 0.0042 & 22 & 0.0240 & -0.0117 & 0.0251 & 0.0263 \\
\hline 23 & 0.0001 & 0.0327 & -0.0076 & -0.0080 & 23 & 0.0283 & \begin{tabular}{l|l}
3 & 0.0136
\end{tabular} & 0.0175 & 0.0049 & 23 & 0.0247 & -0.0115 & 0.0254 & 0.0266 \\
\hline 24 & 0.0001 & 0.0327 & -0.0080 & -0.0076 & 24 & 0.0290 & \begin{tabular}{l|l}
0.0138 \\
\end{tabular} & 0.0173 & 0.0056 & 24 & 0.0253 & -0.0112 & 0.0257 & 0.0269 \\
\hline
\end{tabular}




\section{Referencias}

[1] Argáez Sosa, J. (2014). Un paseo por el modelo GARCH y sus variantes. Abstraction \& Application, 10, 35-50. https://intranet.matematicas.uady.mx/journal/index.php

[2] Baharumshah, A. Z., Sirag, A., \& Soon, S. V. (2017). Asymmetric exchange rate Pass-Through in an emerging market economy: The case of Mexico. Research in International Business and Finance, 41, 247-259. https: //doi.org/10.1016/j.ribaf.2017.04.034

[3] Bailliu, J. y Fuji, E., (2004). Exchange Rate Pass-Through and the Inflation Environment in Industrialized Countries: An Empirical Investigation. Society for Computational Economics, No. 135. https://doi.org/10. 2139/ssrn. 560762

[4] Banco de México (2008, 2009, 2015, 2016). Programa Monetario, 2008, 2009, 2015, 2016, México, varias ediciones. http://www.anterior.banxico.org.mx/publicaciones-y-discursos/publicaciones/ informes-periodicos/politica-monetaria-prog-anual/indexpage.html

[5] Betts, C., y Deveraux, M. (1996). The exchange rate in a model of pricing-to-market. European Economic Review, 40 (3-5), 1007-1021. https://doi.org/10.1016/0014-2921(95)00110-7

[6] Blundell-Wignall, A. (1992). Inflation, Disinflation and Monetary Policy. Economic Group, Reserve Bank of Australia. https://www.rba.gov.au/publications/confs/1992/

[7] Bollerslev, T. (1986). Generalized autoregressive conditional heteroscedasticity. Journal of Econometrics, 31(3), 307-327. https://doi.org/10.1016/0304-4076(86)90063-1

[8] Ca’ Zorzi, M., Hahn, E., y Sánchez, M. (2007). Exchange rate Pass-Through in emerging markets. European Central Bank Working Paper, No. 739. https://www.ecb.europa.eu/pub/pdf/scpwps/ecbwp739.pdf

[9] Capistrán, C., Ibarra, R., y Francia, M. R. (2012). El traspaso de movimientos del tipo de cambio a los precios: un análisis para la economía mexicana. El Trimestre Económico, 79(4), 813. https://doi.org/10.20430/ete. v79i316. 77

[10] Capistrán, C., Ibarra-Ramírez, R., y Ramos-Francia, M. (2011). El traspaso de movimientos del tipo de cambio a los precios: un análisis para la economía mexicana. (No. 2011-12). Working Papers, Banco de México. https: //doi.org/10.20430/ete.v79i316.77

[11] Castillo P, Ramon A., Varela L. Rogelio, y Ocegueda H. J. M. (2013). Traspaso del tipo de cambio al nivel de precios: Un análisis regional. Economía. Sociedad y Territorio, 13(43), 675-695. https://doi.org/10.22136/ est00201343

[12] Choudhri, E. U., Faruqee, H., y Hakura, D. S. (2005). Explaining the exchange rate Pass-Through in different prices. Journal of International Economics, 65(2), 349-374. https://doi.org/10.1016/j.jinteco.2004.02.004

[13] Cortés Espada, J. F. (2013). Estimating the Exchange Rate Pass-Through to Prices in Mexico. Monetaria, XXXV (2), 287-316. https://www.cemla.org/PDF/monetaria/PUB_MON_XXXV-02.pdf

[14] Cruz Aké, S., García Ruiz, R. S., y Venegas-Martínez, F. (2015). Medición no lineal de la dependencia de la inflación sobre el tipo de cambio nominal (Pass-Through). El Trimestre Económico, 82(325), 211-244. https: //doi.org/10.20430/ete.v82i325.145

[15] Cuevas Ahumada, V. M. (2013). La crisis hipotecaria sub-prime y sus efectos sobre México. Análisis Económico, 28(67). http://www.analisiseconomico.azc.uam.mx/index.php/rae/article/view/170

[16] Cuevas Ahumada, V. M. y Sánchez Daza, A. (2012). El efecto traspaso del tipo de cambio a la producción y los precios en América Latina: un enfoque de datos de panel. Calderón, C. y Cuevas, V.M. (Ed.), Macroeconomía abierta en América Latina: teoría y evidencia empírica, 183-210, México: Eón, UAM. https://doi.org/10. 26439/ulima.tesis/6971

[17] Devereux, M. B. y Engel, C. (2002). Exchange rate Pass-Through, exchange rate volatility, and exchange rate disconnect. Journal of Monetary economics, 49(5), 913-940. https: //doi .org/10.1016/s0304-3932(02)00130-7

[18] Dornbusch, R. (1985). Exchange rates and prices. National Bureau of Economic Research. https://core.ac. uk/download/pdf/6822909.pdf 
[19] Dwyer, J., Kent, C. y Pease, A. (1993). Exchange rate Pass-Through: the different responses of importers and exporters. Economic Research Department, Reserve Bank of Australia. https://pdfs.semanticscholar.org/ 4dcd/9249d5dd4af6c98ec971af6f10c4ec2e414f .pdf

[20] Edwards, S. (2006). The relationship between exchange rates and inflation targeting revisited. National Bureau of Economic Research, No. 12163. (DOI): 10.3386/w12163

[21] Federico, K., \& Daniel, S. (2016). Price-Setting and Exchange Rate Pass-Through in the Mexican Economy: Evidence from CPI Micro Data (No. 2016-13). http://www.banxico.org.mx/publications-and-press/ banco-de-mexico-working-papers/\%7BD03AD593-5FDF-AB9D-A240-D30918611135\%7D.pdf

[22] Frankel J., Parsley D. and Shang-Jin, W. (2012). Slow Pass-Through Around the World: A New Import for Developing Countries? Open Economies Review, Springer, 23(2), 213-251. https://doi.org/10.1007/ s11079-011-9210-8

[23] Gagnon, J. E., y Ihrig, J. (2004). Monetary policy and exchange rate pass-through. International Journal of Finance and Economics, 9(4),315-338. https://doi.org/10.1002/ijfe.253

[24] Goldberg, P. K., y Knetter, M. M. (1996). Goods prices and exchange rates: What have we learned? National Bureau of Economic Research, No. w5862. https://doi.org/10.3386/w5862

[25] Goldfajn, I. y S. Werlang (2000). The Pass-Through from depreciation to inflation: A panel study. Banco Central do Brasil, Working Paper, 5. https://www.bcb.gov.br/pec/wps/ingl/wps05.pdf

[26] Gopinath, G., y Rigobon R. (2008). Sticky Borders, Quaterly Journal of Economics, 123 (2), 531-575. https: //doi.org/10.1162/qjec.2008.123.2.531

[27] Guerra, M. (2017). Monetary Policy and the Propagation of Shocks Under Imperfect Exchange-Rate PassThrough. Sobre México. Revista de Economía, 3(3), 46-65. http://www.sobremexico.mx/revista/articles. php?dl=19:021f59

[28] Hahn, E. (2003). Pass-Through of external shocks to euro area inflation. European Central Bank Working Paper Series No. 243. https://www.ecb.europa.eu/pub/pdf/scpwps/ecbwp243.pdf? c2b2d3c1fb18783fdc04fd648f55a5a9

[29] Hernández, J. (2015). Revisión de algunas estimaciones recientes del traspaso del tipo de cambio a los precios en México. Ensayos sobre Política Económica, 33(78), 190-196. https://doi.org/10.1016/j.espe.2015.09.002

[30] Johansen, S. (1991). Estimation and hypothesis testing of cointegration vectors in Gaussian vector autoregressive models. Econometrica: Journal of the Econometric Society, 1551-1580. https://doi.org/10.2307/2938278

[31] Krugman, P. (1986). pricing to market when the exchange rate changes. National Bureau of Economic Research. (DOI): $10.3386 /$ w1926

[32] Mann C. (1989). The effects of exchange rate trends and volatility on export prices: Industry examples from Japan, Germany, and the United States. Review of World Economics, 125 (3), Page 588. https://doi.org/10. $1007 /$ bf02707669

[33] McCarthy, J. (1999). Pass-Through of exchange rates and import prices to domestic inflation in some industrialized economies. Bank for International Settlements, Working papers No. 79. https://www.bis.org/publ/ work79.pdf

[34] Miller, S. (2003). Estimación del Pass-Through del tipo de cambio a precios: 1995-2002. Estudios Económicos, 10. http://www.bcrp.gob.pe/docs/Publicaciones/Revista-Estudios-Economicos/10/ Estudios-Economicos-10-5.pdf

[35] Mishkin, F. S., y Savastano, M. A. (2001). Monetary policy strategies for Latin America. Journal of Development Economics, 66(2), 415-444. https://doi.org/10.1016/s0304-3878(01)00169-9

[36] Ocegueda, J., Torres V., y Manzanares, J. (2011). Tipo de cambio, precios de importación y precios al consumidor: un análisis del efecto de traspaso en México, Quantitativa, 1(1), 105-137. https://doi.org/10.22136/ est00201343

[37] Pérez Laurrabaquio, O. (2012). El traspaso del tipo de cambio a los precios: una aproximación al caso de México, Economía, 376. https://doi.org/10.20430/ete.v79i316.77 
[38] Perron, P. (1989). The Great Crash, the Oil Price Shock, and the Unit Root Hypothesis. Econometrica, No. 57, 1361-1401. https://doi.org/10.2307/1913712

[39] Rodríguez Brindis, M. (2015). Tipo de cambio e inflación en México: evidencia del mecanismo de transmisión de precios. Equilibrio Económico, 11(39), 41-64. https://doi.org/10.2139/ssrn. 2590685

[40] Rodríguez Carranza, J. (2018). Volatilidad cambiaria y el efecto Pass-Through sobre los precios en México. (Tesis de maestría) El Colegio de la Frontera Norte, Tijuana, Baja California. https://doi.org/10.17711/sm. 0185-3325.2018.025

[41] Shioji, E. (2012). The Evolution of the Exchange Rate Pass-Through in Japan: A Re-evaluation Based on TimeVarying Parameter VARs. Public Policy Review, Policy Research Institute, Ministry of Finance Japan, 8(1), 67-92. https://ideas.repec.org/a/mof/journl/ppr015d.html

[42] Solorzano, J. D. (2017). Heterogeneous Exchange Rate Pass-Through in Mexico: What Drives It? https://pdfs . semanticscholar.org/504e/0b439bca180b5b4b4daa632807af40e541e6.pdf

[43] Stulz, J. (2007). Exchange Rate Pass-Through in Switzerland: Evidence from Vector Autorregressions. Swiss National Bank Economic Studies No.4. https://pdfs.semanticscholar.org/65c4/ b4d36edaf4be5ec155d7dde1be601876fbb5.pdf

[44] Taylor, J. B. (2000). Low inflation, Pass-Through, and the pricing power of firms. European Economic Review, 44(7), 1389-1408. https://doi.org/10.1016/s0014-2921(00)00037-4

[45] Wei, S. J., y Parsley, D. C. (1995). Purchasing power disparity during the floating rate period: exchange rate volatility, trade barriers and other culprits. National Bureau of Economic Research, No. 5032. https://doi .org/ $10.3386 /$ w5032 